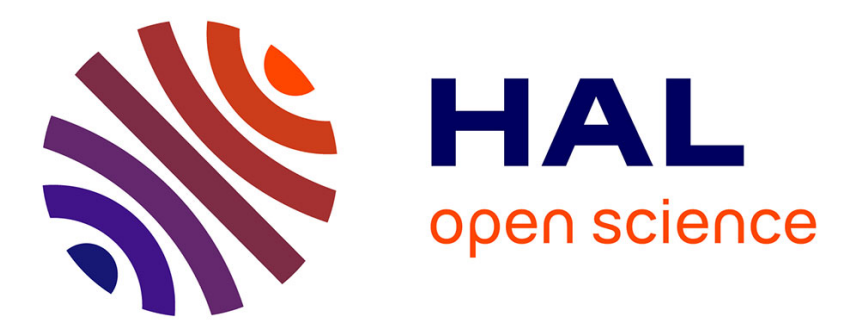

\title{
Obtention de la vacuité utérine dans le cadre d'une perte de grossesse
}

\author{
Gaël Beucher, Patricia Dolley, Zelda Stewart, Vincent Lavoué, Xavier
}

Deffieux, Michel Dreyfus

\section{- To cite this version:}

Gaël Beucher, Patricia Dolley, Zelda Stewart, Vincent Lavoué, Xavier Deffieux, et al.. Obtention de la vacuité utérine dans le cadre d'une perte de grossesse. Journal de Gynécologie Obstétrique et Biologie de la Reproduction, 2015, in press. 10.1016/j.jgyn.2014.09.013 . hal-01102796

\section{HAL Id: hal-01102796 \\ https://hal.science/hal-01102796}

Submitted on 13 Jan 2015

HAL is a multi-disciplinary open access archive for the deposit and dissemination of scientific research documents, whether they are published or not. The documents may come from teaching and research institutions in France or abroad, or from public or private research centers.
L'archive ouverte pluridisciplinaire HAL, est destinée au dépôt et à la diffusion de documents scientifiques de niveau recherche, publiés ou non, émanant des établissements d'enseignement et de recherche français ou étrangers, des laboratoires publics ou privés. 


\section{Obtention de la vacuité utérine dans le cadre d'une perte de grossesse}

Obtaining of uterine vacuity in pregnancy loss

Gaël Beucher ${ }^{\mathbf{a} *}$, Patricia Dolley ${ }^{\mathbf{a}}$, Zelda Stewart ${ }^{\mathbf{a}, \mathbf{b}}$, Vincent Lavoué ${ }^{\mathbf{c}}$, Xavier Deffieux ${ }^{\mathbf{d}, \mathbf{e}}$, Michel Dreyfus a,b

a Service de Gynécologie Obstétrique et Médecine de la Reproduction, CHU de Caen, avenue Côte de Nacre, 14033 Caen Cedex 9, France.

b UFR de Médecine, Université de Caen Basse Normandie, avenue Côte de Nacre, 14033 Caen Cedex 9, France.

' Service de gynécologie-obstétrique, CHU Anne-de-Bretagne, 16, boulevard de Bulgarie, BP 90347, 35203 Rennes cedex 2.

d Service de gynécologie-obstétrique et médecine de la reproduction, hôpital Antoine-Béclère, AP-HP, 157, rue de la Porte-de-Trivaux, 92140 Clamart, France

${ }^{\text {e }}$ Faculté de médecine, Université Paris-Sud, 91405 Orsay, France.

* Auteur correspondant : même adresse

Tel : 02-31-27-20-40 ; Fax : 02-31-27-26-28

Adresse e-mail : beucher-g@chu-caen.fr

Evacuation utérine en cas de perte de grossesse 


\section{Résumé}

Objectif : Evaluer les bénéfices et les risques précoces et tardifs des traitements des fausses couches (FC) précoces et des méthodes d'induction du travail et d'évacuation utérine en cas de mort fotale in utero (MFIU) au-delà du premier trimestre de la grossesse.

Méthode: Recherche bibliographique en langues française et anglaise effectuée par consultation des banques de données PubMed et Cochrane Library.

\section{Résultats :}

En cas de grossesse arrêtée, l'expectative n'est pas recommandée (Grade A) car elle augmente les risques d'absence d'expulsion spontanée nécessitant un traitement chirurgical non programmé et de transfusion sanguine (NP 1). Le traitement par aspiration d'une grossesse arrêtée est plus efficace que le traitement par misoprostol (NP 1), mais les techniques médicale et chirurgicale comportent des risques de complications rares et comparables (NP1). Lorsqu'un traitement médical a été choisi, le misoprostol doit être administré par voie vaginale à la dose unique de $800 \mu \mathrm{g}$, éventuellement renouvelée au bout de 24-48 heures (Grade B). L'administration préalable de mifépristone n'est pas recommandée (Grade B).

En cas de FC incomplète, l'expectative peut être proposée en première intention (Grade A). Le traitement par misoprostol n'est pas recommandé (Grade B) car il n'améliore pas le taux d'évacuation par rapport à une simple expectative et ne réduit pas les risques de complications (NP 2). L'aspiration permet des taux d'évacuation complète élevés (97-98\%) pour des risques de complications hémorragiques et infectieuses faibles $(<5 \%)(\mathrm{NP} 1)$. Toutefois, il ne doit pas être imposé à la patiente en raison de la bonne efficacité (plus de $75 \%$ ) et des risques de complications rares de l'expectative (Grade A). L'aspiration chirurgicale doit être préférée au curetage car elle est plus rapide, moins douloureuse et moins hémorragique (Grade B). La fertilité ultérieure est identique quel que soit le traitement d'une FC précoce (NP 2). En cas de 
suspicion de rétention trophoblastique prolongée, une hystéroscopie diagnostique est recommandée (Grade B).

En cas de MFIU, le protocole d'administration du misoprostol le plus efficace en l'absence d'utérus cicatriciel est la dose de 200 à $400 \mu \mathrm{g}$ par voie vaginale, renouvelée toutes les 4 à 6 heures (Grade B). L'administration préalable d'une dose de $200 \mathrm{mg}$ de mifépristone au moins 24 heures avant celle de misoprostol est recommandée (Grade C) car elle améliore les délais d'expulsion et diminue les besoins en misoprostol (NP 3).

Mots-clés : Fausse couche précoce, misoprostol, aspiration utérine chirurgicale, perte fœtale, mort fœetale, induction du travail. 


\begin{abstract}
Objective: To assess early and late benefits and harms of different management options for first-trimester miscarriage and for induction of labor and obtaining of uterine vacuity in case of fetal death beyond of 14 weeks of gestation.
\end{abstract}

Methods: French and English publications were searched using PubMed and Cochrane Library.

\title{
Results:
}

Concerning missed miscarriage, expectant management is not recommended (LE 1) because it increases the risk of failure, need of unplanned surgical procedure and blood transfusion (LE 1). Surgical uterine evacuation remains more effective than medical treatment using misoprostol (LE 1), but both techniques involve rare and comparable risks (EL 1). When chosen, medical treatment should be a vaginal dose of $800 \mu \mathrm{g}$ of misoprostol, possibly repeated 24 to 48 hours later (EL 2). Administration of mifepristone prior to misoprostol is not recommended (EL 2).

In case of incomplete miscarriage, expectant management can be offered because it does not increase the risk of complications, neither haemorrhagic nor infectious (EL 1). Medical treatment using misoprostol is not recommended (EL 2) because it does not improve the evacuation rate when compared to our first option, and does not reduce the risk of complications (EL 2). Surgical uterine evacuation leads to high evacuation rate (97-98\%) and low risk of complications, haemorrhagic and infectious $(<5 \%)$ (EL1). However this option should not be the only one because of the good efficiency of the expectant management (more than $75 \%$ of evacuation) and comparably low risk of complications (EL 1). Surgical aspiration should be favoured to curettage because it is quicker, less painful and leads to less bleeding (EL 2). After a first trimester miscarriage future fertility is identical with each 
treatment (EL 2). When a trophoblastic retention is suspected, a diagnostic hysteroscopy is recommended (EL 2).

In case of late intrauterine foetal death beyond 14 weeks of gestation and without a past caesarean section, the most efficient protocol seems to be vaginal administration of misoprostol 200 to $400 \mu \mathrm{g}$ every 4 to 6 hours (LE 2). 24 hours prior to misoprostol the administration of $200 \mathrm{mg}$ of mifepristone is recommanded (EL 3) because it improves the induction-expulsion time and diminishes the quantity of needed misoprostol (and so the complications linked to it) (EL 3).

Key words: Early miscarriage, early pregnancy failure, misoprostol, chirurgical uterine evacuation, fetal loss, pregnancy loss, late intrauterine fetal death, stillbirth, pregnancy termination, labor induction. 
L'objectif de ce travail était d'évaluer les bénéfices et les risques à court et long terme des différentes méthodes pour provoquer l'expulsion du contenu utérin (traitement chirurgical, traitement médical et expectative) en fonction du stade évolutif de la fausse couche (FC) et de l'âge gestationnel de la mort fotale in utero (MFIU).

\section{Méthodologie}

La recherche bibliographique a été effectuée à l'aide des bases de données informatiques Medline et de la Cochrane Library entre 1990 et mai 2014. Les mots clés suivants ont été choisis et combinés en autant d'étapes que nécessaire en fonction de l'âge gestationnel. Premier trimestre de la grossesse: early miscarriage, first trimester miscarriage, early pregnancy failure, early pregnancy loss, misoprostol, mifepristone, surgical evacuation, cervical preparation, cervical ripening, intrauterine adhesions, infertility, reproductive outcome. Deuxième et troisième trimestres: fetal demise, late intrauterine fetal death, stillbirth, second-trimester and third trimester abortion, induced abortion, labor termination, labor induction. Seules ont été retenues les publications de langues anglaise et française. Celles-ci ont été classées par niveau de preuve. Plusieurs revues de synthèse sur le sujet ont été consultées. La recherche informatisée a été complétée par une recherche manuelle des références des articles sélectionnés.

\section{Evacuation utérine d'une FC du premier trimestre}

\section{Grossesse arrêtée au premier trimestre}

Plusieurs méta-analyses ont comparé les bénéfices et les risques des différentes alternatives thérapeutiques en cas de grossesse arrêtée précoce : l'évacuation chirurgicale du produit de FC, le traitement médical par le misoprostol et l'expectative [1-4]. Les études randomisées incluses avaient une méthodologie différente en termes de critères d'inclusion, de modalités 
d'administration du misoprostol (posologie et voie d'administration) et de critères de jugement (définition du succès de la méthode, complications). Certaines incluaient sans distinction dans la même analyse des grossesses arrêtées et des FC incomplètes [4]. La méthodologie et les résultats des principales méta-analyses [1-4] et études randomisées [5, 6] sont résumés dans le tableau 1. Les avantages (bénéfices) et les inconvénients (risques) de chaque prise en charge thérapeutique (chirurgicale ou médicale) sont synthétisés dans le

\section{tableau 2.}

Le succès (ou l'efficacité) d'une méthode thérapeutique est habituellement jugé sur la réalisation d'une évacuation complète du contenu utérin dans un délai préalablement déterminé, sans recours à une évacuation chirurgicale secondaire non programmée et en l'absence de complications à court terme, essentiellement d'hémorragie et d'infection.

\section{Expectative en cas de grossesse arrêtée asymptomatique}

L'évolution naturelle d'une FC du premier trimestre aboutit dans plus de $75 \%$ des cas à l'expulsion spontanée et complète sans majoration des risques hémorragiques ou infectieux par rapport aux autres alternatives thérapeutiques (médicale et chirurgicale) $[2,4,5,7](\mathrm{NP}$ 1). Cependant, les délais d'expulsion sont souvent prolongés (2 à 6 semaines) et inacceptables pour les patientes, avec des risques élevés (28 à $80 \%$ ) de consultation et de geste chirurgical non programmé (pour persistance d'une rétention ou à la demande de la patiente) $[\mathbf{2 - 5}, \mathbf{7}, \mathbf{8}]$ (NP 1).

Dans une méta-analyse de la Cochrane Database ayant comparé le misoprostol par voie vaginale (800 $\mu \mathrm{g}$ éventuellement renouvelés à 24 heures) à un placebo, le taux d'évacuation complète à 7 jours (une étude, 83 femmes) et le recours à un traitement chirurgical (une étude, 104 femmes) étaient respectivement de 86 versus $29 \%$ (RR 3, IC $95 \%$ 1,8-5) et 32 versus $82 \%$ (RR 0,4 IC $95 \%$ 0,26-0,6) [3] (NP 2). Par contre, les risques de complications 
hémorragiques (besoin en transfusion et saignements durant plus de deux semaines) et infectieuses étaient comparables entre les deux groupes.

Dans une autre méta-analyse de la Cochrane Database ayant comparé une simple expectative au traitement chirurgical, les risques d'évacuation incomplète étaient significativement augmentés à deux semaines (quatre études, 1263 femmes, RR 3,98 IC $95 \%$ 2,94-5,38) et dans un délai de six à huit semaines (trois études, 430 femmes, RR 2,56 IC $95 \%$ 1,15-5,69) en cas d'expectative par rapport au traitement chirurgical par aspiration immédiate [7] (NP 1). En cas d'expectative, le risque d'avoir recours à un traitement chirurgical non programmé était significativement augmenté (cinq études, 1454 femmes, 28 versus $4 \%$, RR 7,35 IC $95 \%$ 5,04-10,72) par rapport au traitement chirurgical (NP 1). Le besoin en transfusion sanguine était également significativement augmenté (trois études, 1205 femmes, 1,4\% versus 0, RR 6,45 IC $95 \%$ 1,21-34,42) (NP 1). Le risque d'infection du post-abortum était similaire entre les deux groupes (0 à $10 \%$, sept études 1514 femmes) [7] (NP 1). Une étude randomisée française ayant inclus 182 grossesses arrêtées du premier trimestre a montré qu'un délai d'expectative d'une semaine entre le diagnostic et le début du protocole médical (mifépristone $200 \mathrm{mg}$ par voie orale puis misoprostol $400 \mu \mathrm{g}$ par voie vaginale deux jours après) exposait à une augmentation significative des risques d'aspiration chirurgicale (43,5 versus $19 \%, \mathrm{p}<0,001)$, notamment effectuée en urgence (20 versus 4,5\%, p=0,001) mais aussi de consultations d'urgence (34 versus $17 \%, \mathrm{p}=0,009)$ [9] (NP 2).

Au total, l'expectative en cas de grossesse arrêtée augmente les risques d'absence d'expulsion spontanée nécessitant un traitement chirurgical non programmé et de transfusion sanguine (NP 1). En cas de grossesse arrêtée, l'expectative n'est pas recommandée (Grade A).

Traitement chirurgical en cas de grossesse arrêtée asymptomatique 


\section{Efficacité du traitement chirurgical}

Les taux de succès (évacuation complète sans geste complémentaire) rapportés dans les études sont élevés, compris entre 95 et $98 \%$ (tableau 2) [2, 5, 6] (NP 1). Ils demeurent significativement plus élevés que ceux obtenus après un traitement médical par misoprostol, quelles que soient ses modalités d'administration [2-4] (NP 1).

\section{Complications à court terme du traitement chirurgical}

En cas de traitement chirurgical, les risques de complications à court terme sont rares : infection utérine ( 0 à $6 \%$ ), hémorragie excessive nécessitant un traitement chirurgical complémentaire et/ou une transfusion sanguine ( 0 à $3 \%$ ), déchirure cervicale ou perforation utérine nécessitant un traitement chirurgical complémentaire (0 à $2 \%)$, décès $(0,5 / 100000)$

\section{$[1,2,5,6](N P 1)$.}

Prévention des complications à court terme du traitement chirurgical

En 2014, un état des connaissances sur la préparation cervicale avant évacuation chirurgicale du contenu utérin au premier trimestre de la grossesse a été réalisé par le Collège National des Gynécologues Obstétriciens Français (CNGOF) [10]. Aucune étude n'avait évaluée spécifiquement la préparation cervicale avant un traitement chirurgical pour une FC précoce. En cas de grossesse encore évolutive, les conclusions de cet état des connaissances étaient les suivantes. La préparation cervicale préalable est efficace pour faciliter la dilatation cervicale, diminuer la durée opératoire et le risque de rétention utérine secondaire (NP 1). Le misoprostol (400 $\mu \mathrm{g}$ administrés par voie sublinguale ou vaginale, respectivement 2 et 3 heures avant la procédure chirurgicale), la mifépristone (200 mg par voie orale 24 heures avant la procédure) ou les dilatateurs osmotiques mis en place la veille de la procédure sont des alternatives équivalentes en terme d'efficacité et de tolérance [10] (NP 2). La réduction du risque de traumatisme opératoire (perforation utérine et déchirure cervicale) n'a pas été démontrée par les études comparatives mais il s'agit d'évènements rares $(0$ à $2 \%)[\mathbf{2}, \mathbf{3}, \mathbf{5}]$. 
Dans les études, l'évacuation chirurgicale du contenu utérin est réalisée essentiellement au bloc opératoire par une aspiration motorisée à l'aide d'une canule [6]. Il n'existe pas à notre connaissance d'essais ayant comparé l'évacuation d'une grossesse arrêtée par aspiration et par curetage à l'aide d'une curette. L'aspiration peut aussi être réalisée de façon manuelle à l'aide d'une canule reliée à une seringue. Une méta-analyse a colligé quatre études de faible qualité méthodologique (une seule était randomisée) ayant comparé ces deux techniques (aspirations motorisée et manuelle) en cas de FC du premier trimestre [1]. Celles-ci étaient équivalentes en termes d'efficacité, d'infections et de satisfaction des patientes. En revanche, les douleurs dans les 48 heures et les saignements nécessitant une transfusion sanguine étaient significativement plus fréquents après aspiration manuelle [1] (NP 3).

En cas de traitement chirurgical d'une FC incomplète, plusieurs études randomisées de faible qualité méthodologique n'ont pas montré de diminution significative du risque d'infection du post-abortum en réalisant une antibioprophylaxie peropératoire par doxycycline ou céfoxitine $[11,12](N P 2)$.

Il n'existe pas d'étude randomisée ayant évalué l'intérêt du guidage échographique peropératoire et du contrôle de la vacuité utérine lors du traitement chirurgical d'une FC du premier trimestre afin de réduire les risques de complications opératoires immédiates (traumatismes, hémorragies) et secondaires (infections, rétentions).

L'intérêt de l'administration d'oxytocine après une aspiration chirurgicale d'une FC du premier trimestre dans le but de diminuer la survenue de complications hémorragiques n'a pas été évalué.

Au total, le traitement chirurgical par aspiration d'une grossesse arrêtée est plus efficace que le traitement médical par le misoprostol (NP 1). Les risques de complications à court terme, traumatiques, hémorragiques ou infectieuses sont rares (moins de cinq pour cent) (NP 1). 


\section{Traitement médical par le misoprostol en cas de grossesse arrêtée asymptomatique}

\section{Efficacité en fonction des modalités d'administration du misoprostol}

L'efficacité du misoprostol à provoquer l'expulsion du produit de conception sans recours au geste chirurgical dépend largement du mode d'administration, de la dose administrée et du délai entre le début de la procédure et la décision de son échec [1, 3].

En 2014, un état des connaissances sur l'utilisation du misoprostol hors AMM en cas de FC du premier trimestre a été réalisé par le CNGOF [10].

Dans une méta-analyse de la Cochrane Database, l'administration orale était moins efficace que par voie vaginale avec des taux de succès respectifs de 82 et $87 \%$ (2 études, 218 femmes, RR 0,90 IC $95 \%$ 0,82-0,99) mais les doses n'étaient pas identiques entre les deux voies d'administration dans une étude (respectivement 400 et $800 \mu \mathrm{g}$ ) [3] (NP 2). La tolérance digestive, l'intensité des douleurs, la survenue de fièvre et le degré de satisfaction des patientes étaient toutefois semblables entre les deux voies d'administration. Par contre, le délai moyen entre la prise médicamenteuse et l'expulsion était significativement plus court par voie vaginale (une étude, 70 femmes, 10 versus 15 heures, $p<0,00001$ ) [3] (NP 2). L'administration d'une dose vaginale de $800 \mu \mathrm{g}$, renouvelée si besoin à 24 ou 48 heures, permet le plus souvent d'obtenir un taux d'expulsion complète supérieur à $80 \%[\mathbf{2 , 3 , 6 , 1 3}$ (NP 1). Cette dose de $800 \mu \mathrm{g}$ a tendance à être plus efficace que l'utilisation de $400 \mathrm{ou} 600 \mu \mathrm{g}$ (3 études, 247 femmes, 74 versus $63 \%$, RR 0,85 IC $95 \%$ 0,72-1) sans augmenter les risques d'effets secondaires (fièvre et troubles digestifs) [3] (NP 2). Elle a cependant besoin d'être renouvelée dans au moins $30 \%$ des cas au bout de 24-48 heures en l'absence d'expulsion du sac gestationnel (SG) [6, 13] (NP 2). Elle est néanmoins aussi efficace que des doses de 200 à $400 \mu \mathrm{g}$ répétées à intervalle régulier de 3 à 4 heures $[3,13]$ (NP 2). L'efficacité de l'administration vaginale n'est pas améliorée par l'humidification ou le fractionnement des 
comprimés [3] (NP 2). L'administration sublinguale de $600 \mu \mathrm{g}$ répétée une à deux fois toutes les 3 heures a une efficacité équivalente à celle de la voie vaginale utilisée selon le même protocole (taux de succès de 87,5\%) mais des effets secondaires digestifs plus fréquents (diarrhées) (1 étude, 80 femmes) [14] (NP 2). La poursuite d'une administration sublinguale pendant une semaine n'améliore pas le taux de succès mais augmente significativement les effets secondaires digestifs [15] (NP 2).

Les chances de succès (expulsion du SG) augmentent avec le délai entre l'administration et la décision d'échec du traitement médical (8 à 72 heures versus 7 à 15 jours) sans majoration des risques de complications (geste chirurgical en urgence, infection) $[\mathbf{5}, \mathbf{6}, \mathbf{1 3}]$ (NP 2). Les risques d'échec ou de complications du traitement médical ne semblent corrélés ni à la taille du SG (diamètre moyen entre 16 et $50 \mathrm{~mm}$ ) ni à celle de l'embryon (longueur cranio-caudale entre 5 et $45 \mathrm{~mm}$ ) $[\mathbf{6}, \mathbf{1 6}]$ (NP 2). A l'inverse, les facteurs prédictifs significatifs du succès de la méthode sont une faible parité, l'existence préalable ou dans les 24 heures de douleurs et de saignements, un col ouvert ou la présence de matériel dans le col dans les 48 heures (valeur prédictive positive supérieure à $80 \%)[\mathbf{1 7}, \mathbf{1 8}]$ (NP 2). L'expulsion du SG confirmée par la réalisation d'une échographie endovaginale après un délai suffisant (24-48 heures) semble être le meilleur critère pour juger du succès de la méthode médicale [19] (NP 3). L'évaluation de la mesure antéro-postérieure des images intra-utérines réduit les taux de succès et induit une augmentation du risque de traitement chirurgical complémentaire [19] (NP 3). L'administration préalable de mifépristone (200 mg 24 à 48 heures avant) pour potentialiser l'efficacité du misoprostol n'améliore pas les taux de succès par rapport à l'usage seul du misoprostol [3, 9, 20-22] (NP 2).

Innocuité et tolérance du traitement médical par le misoprostol 
Les résultats publiés sont en faveur de l'innocuité du misoprostol avec des risques de complications identiques à ceux du traitement chirurgical : geste chirurgical en urgence pour hémorragie (0 à $5 \%)$, infection du post-abortum (0 à $3 \%)[\mathbf{1 - 3 , 5 , 6 ]}$ (Tableau 1) (NP 1).

Le rapport bénéfice-risque semble donc largement favorable à l'administration vaginale du misoprostol (NP 1). Les effets secondaires (nausées, vomissements, diarrhées, fièvre, frissons) semblent significativement moins fréquents après administration vaginale qu'orale ou sublinguale et sont le plus souvent bien tolérés et transitoires [1, 3] (NP 2). Ils sont rapportés dans 15 à $40 \%$ des cas quelles que soient les modalités d'administration du misoprostol [2]. Les effets secondaires digestifs sont également plus fréquents qu'après un traitement chirurgical, quels que soient le mode d'administration et la dose de misoprostol (tableau 1) [1] (NP 1). Après un traitement médical, la durée moyenne des saignements est de 7 à 15 jours, significativement plus longue qu'après un geste chirurgical $(p<0,0004)[\mathbf{1}, \mathbf{5}$, 20, 23] (NP 1). L'abondance des saignements est également plus importante avec le traitement médical, avec une augmentation significative du delta d'hémoglobine calculé à deux semaines $(-0,7$ versus $-0,2 \mathrm{~g} / \mathrm{dl}, \mathrm{p}<0,001)$ et de la durée des saignements jugés abondants (au moins deux garnitures par heure, 4 jours versus 1 jour $\mathrm{p}<0,001$ ) [23] (NP 2). Dans cette analyse secondaire de l'essai randomisé de Zhang et al, il existait une corrélation positive après analyse multivariée entre l'âge gestationnel de la FC $(7,6 \mathrm{SA}$ +/-1,5 en moyenne) et l'importance des variations des taux d'hémoglobine entre l'inclusion et à deux semaines [23] (NP 2). De même, les douleurs pelviennes sont plus fréquentes et jugées plus importantes $(\mathrm{p}<0,001)[\mathbf{1}, \mathbf{6}]$, requérant plus souvent la prise d'antalgiques $[\mathbf{1}, \mathbf{5}](\mathrm{NP} 1)$. Les antalgiques mineurs (paracétamol, anti-inflammatoires non stéroïdiens), parfois associés à ceux de deuxième palier, sont le plus souvent suffisants au moment de l'expulsion $[\mathbf{6 , 1 3}$ (NP 2). 
Le nombre de femmes ayant eu besoin d'une consultation en urgence était statistiquement comparable après le traitement médical et la chirurgie (tableau 2) [1] (NP 1). Par contre, le taux d'hospitalisations non programmées était plus élevé en cas de traitement médical (tableau 1) [5] (NP 2).

L'efficacité et l'innocuité d'une dose vaginale de $800 \mu \mathrm{g}$, éventuellement renouvelée, ne sont pas altérées en cas d'utérus cicatriciel (analyse secondaire d'un essai randomisé) [24] (NP 2).

\section{Satisfaction des patientes}

L'indice de satisfaction des patientes traitées médicalement est généralement excellent (supérieur à 80 \%) et demeure comparable à celui des femmes traitées chirurgicalement $[\mathbf{1}, \mathbf{3}]$ (NP 2). Il est cependant corrélé au succès ou non de la méthode et à l'importance des douleurs

[3] (NP 2). Dans l'essai anglais de Trinder et al. (MIST Trial), le retentissement sur le vécu psychologique et social des patientes était identique quel que soit le traitement, avec un temps moyen de retour à une vie normale de 2 jours, une durée moyenne de l'arrêt de travail de 8 à 9 jours et des scores de dépression et d'anxiété identiques six à huit semaines après la randomisation [5] (NP 2). Une étude randomisée australienne récente (180 femmes) n'a montré aucune différence sur les scores de satisfaction, de bien-être psychologique, de dépression et d'anxiété après le traitement médical ou chirurgical d'une FC du $1^{\mathrm{er}}$ trimestre [25] (NP 2).

Comparaison médico-économique des traitements chirurgical et médical en cas de grossesse arrêtée

Deux analyses médico-économiques ont été réalisées à partir des résultats de deux essais randomisés ayant comparé le misoprostol administré par voie vaginale au traitement chirurgical [26, 27]. Les critères évalués étaient les hospitalisations, les consultations programmées et effectuées en urgence, les actes d'échographie, de chirurgie et d'anesthésie. Ces deux analyses observaient une diminution significative des coûts en cas de traitement 
médical d'autant plus importante que le taux d'évacuation complète après l'administration du misoprostol était élevé, en raison de la diminution des besoins en chirurgie complémentaire (NP 2). Par contre, le coût du traitement chirurgical devenait significativement moins important en cas d'aspiration manuelle effectuée en ambulatoire et lorsque le besoin de consultations de surveillance augmentait en cas de traitement médical [26] (NP 2).

Au total, en cas de grossesse arrêtée au premier trimestre, les techniques médicale et chirurgicale comportent des risques de complications à court terme, traumatiques, hémorragiques et infectieuses, rares et comparables (moins de cinq pour cent) (NP 1). Le traitement médical par misoprostol est responsable de saignements plus prolongés et de douleurs plus fréquentes que le traitement chirurgical (NP 1). Le risque d'une hospitalisation non programmée est également plus élevé (NP 2). Le recours à une consultation en urgence et le degré de satisfaction des patientes sont comparables entre les deux techniques (NP 2). Les effets secondaires du traitement médical par misoprostol (troubles digestifs, douleurs et fièvre) sont en général bien tolérés (NP 2). Le traitement médical par misoprostol permet de réduire les coûts de la prise en charge des grossesses arrêtées du premier trimestre en diminuant les hospitalisations et les actes d'anesthésie et de chirurgie (NP 2). La patiente doit être informée des avantages et inconvénients de chaque technique. Elle doit également être informée de l'utilisation du misoprostol hors AMM.

Lorsqu'un traitement médical a été choisi, le misoprostol doit être administré par voie vaginale à la dose unique de $800 \mu \mathrm{g}$, éventuellement renouvelée au bout de 24-48 heures (taux de succès le plus souvent supérieur à 80 \%) (Grade B). L'administration préalable de mifépristone n'est pas recommandée (Grade B). En cas de persistance du SG après la prise de misoprostol, il semble raisonnable de respecter un certain délai (deux jours à deux semaines) avant d'envisager une autre alternative thérapeutique (Grade C). 


\section{FC du premier trimestre en cours d'expulsion}

Aucune étude n'a comparé différentes attitudes thérapeutiques (expectative versus évacuation utérine immédiate) dans cette circonstance.

En cas de FC hémorragique nécessitant une évacuation chirurgicale utérine urgente, il n'existe pas d'études prospectives ayant comparé et évalué les différentes alternatives thérapeutiques telles que le tamponnement intra-utérin ou l'embolisation artérielle. L'utilisation d'une sonde de Foley ou de Bakri a été rapportée en cas de saignements excessifs survenant lors d'une évacuation chirurgicale du contenu utérin [28-30] (NP 4). Des courtes séries d'embolisation des artères utérines ont montré des taux de succès de 90 à $100 \%$ dans cette indication [31-33] (NP 4).

\section{FC incomplète au premier trimestre}

\section{Expectative}

En cas de FC incomplète, un délai d'expectative variable de trois jours à huit semaines permet d'obtenir dans 75 à $100 \%$ des cas (critères échographiques) une évacuation complète sans le recours à un geste chirurgical complémentaire $[\mathbf{2}, \mathbf{4 , 5}, \mathbf{8}](\mathrm{NP} 1)$. Dans une série prospective de 203 femmes présentant une FC incomplète, deux semaines d'expectative permettaient d'obtenir un taux de succès de $71 \%$ pour un taux de chirurgie en urgence de $2,5 \%$ [34] (NP 3). Les risques de complications hémorragiques (3 à $6 \%$ ) et infectieuses (1 à $2 \%$ ) ne sont pas augmentés par rapport à une prise en charge chirurgicale immédiate $[\mathbf{2}, \mathbf{4}, \mathbf{5}]$ (NP 1). Les saignements peuvent être abondants les premiers jours après l'expulsion (moins d'une semaine) et peuvent justifier parfois d'une consultation en urgence (dans $30 \%$ des cas) [5] NP 2). Leur durée moyenne est de 7 à 15 jours, sans qu'il y ait de corrélation positive avec 
l'importance des images échographiques intra-utérines (mesure antéropostérieure comprise entre 10 et $30 \mathrm{~mm})[\mathbf{1 9}, \mathbf{3 5}](\mathrm{NP} 3)$.

Un essai randomisé multicentrique hollandais actuellement en cours compare une attitude d'expectative à la réalisation d'un traitement chirurgical en cas d'évacuation utérine incomplète après un traitement médical par le misoprostol [36]. Le critère de jugement principal était l'obtention d'une vacuité utérine échographique à six semaines définie par une mesure intra-utérine maximale inférieure à $10 \mathrm{~mm}$.

Au total, en cas de FC incomplète, une attitude d'expectative permet d'obtenir une évacuation complète de l'utérus dans plus de $75 \%$ des cas sans augmentation des risques de complications hémorragiques et infectieuses (NP1). Elle peut être proposée en première intention (Grade A). Il n'est pas possible de définir une limite à la durée d'expectative car celle-ci varie en fonction des études (de trois jours à huit semaines).

\section{Traitement médical par le misoprostol}

Une méta-analyse de la Cochrane Database a colligé 20 études randomisées (4208 femmes) ayant comparé les différentes alternatives thérapeutiques en cas de FC incomplète du $1^{\text {er }}$ trimestre de la grossesse [37]. Trois essais (335 femmes) comparant le misoprostol administré par voie vaginale $(400$ à $800 \mu \mathrm{g})$ à l'expectative ont été inclus. Les taux d'évacuation complète à deux semaines étaient élevés (supérieurs à $80 \%$ ) et similaires entre les deux groupes (NP 2). Les risques de traitement chirurgical non programmé (respectivement 20 et $30 \%$ ), de complications hémorragiques (1 seule transfusion sanguine dans le groupe misoprostol) et infectieuses (4 versus $1 \%$ ) étaient statistiquement comparables entre le misoprostol et l'expectative (NP 2) [37]. Vingt essais (2894 femmes) ont comparé le traitement chirurgical à l'administration de misoprostol par voir vaginale, orale ou sublinguale. Les taux d'évacuation complète étaient significativement diminués dans le groupe misoprostol par rapport au groupe 
chirurgie (94 versus $98 \%$, RR 0,97 IC $95 \%$ 0,95-0,99) mais demeuraient élevés, compris entre 80 et $99 \%$ (NP 1). Les risques de complications opératoires étaient rares mais significativement diminués dans le groupe misoprostol (moins de $5 \%$, RR 0,06 IC $95 \%$ 0,020,13), s'expliquant par le faible taux de traitement chirurgical secondaire dans ce groupe. Par contre, le risque de geste chirurgical non programmé était significativement augmenté (10 versus $1 \%$, RR 5,82 IC $95 \%$ 2,93-11,56), ainsi que les risques de troubles digestifs (nausées et vomissements) et la durée moyenne des saignements (NP 1). Les risques de transfusion sanguine et d'infection pelvienne (moins de $2 \%$ ), les scores de satisfaction (plus de $90 \%$ ) et les besoins en antalgiques étaient similaires entre les deux groupes. Cinq études colligées dans cette méta-analyse ont comparé différentes modalités d'administration du misoprostol (voie et posologie) sans qu'aucun schéma thérapeutique n'apparaisse supérieur à un autre (taux de succès entre 95 et $100 \%)$ [37] (NP1).

Au total, en cas de FC incomplète, le traitement médical par misoprostol n'améliore pas le taux d'évacuation utérine complète à deux semaines par rapport à une simple expectative et ne réduit pas les risques de complications hémorragiques et infectieuses (NP 2). Il n'est pas recommandé (Grade B).

\section{Traitement chirurgical}

L'évacuation chirurgicale d'une rétention du produit de FC offre des taux de succès élevés (97$98 \%$ ) et de complications hémorragiques et infectieuses faibles (moins de $5 \%)[\mathbf{2}, \mathbf{3}, \mathbf{5}](\mathrm{NP}$ 1). Les risques de consultations non programmées, de geste chirurgical en urgence, de saignements prolongés et abondants sont significativement inférieurs à ceux des autres alternatives [5, 7, 37] (NP 1). Une méta-analyse de la Cochrane Database a inclus deux études randomisées (550 femmes) ayant comparé 1'aspiration chirurgicale au curetage à l'aide d'une curette [38]. L'aspiration chirurgicale à l'aide d'une canule était significativement moins 
hémorragique, plus rapide et moins douloureuse que le simple curetage à la curette. Les risques de complications opératoires ( 0 à $1 \%)$, infectieuses $(1$ à $5 \%)$ et de rétention secondaire (1 à 2 $\%$ ) étaient similaires entre les 2 techniques [38] (NP 2).

En l'absence de données, il est impossible de définir des situations cliniques pour lesquelles le traitement chirurgical s'impose en première intention, en dehors de l'évacuation urgente du contenu utérin. La préférence de la patiente pour une aspiration chirurgicale, méthode efficace, sûre et à faible risque de consultation non programmée doit être prise en compte dans la décision thérapeutique. A l'inverse, le traitement chirurgical ne doit pas être imposé systématiquement en présence d'une image intra-utérine pouvant faire craindre une rétention trophoblastique en raison de l'absence d'augmentation des risques de complications hémorragiques et infectieuses en cas de simple expectative (NP 1).

Au total, en cas de FC incomplète, le traitement chirurgical permet des taux d'évacuation complète élevés (97-98\%) pour des risques de complications hémorragiques et infectieuses faibles (moins de $5 \%$ ) (NP 1). Il permet également de réduire les risques de consultations et de traitement chirurgical non programmés par rapport aux autres alternatives (NP 1). Il ne doit pas être imposé à la patiente en raison de la bonne efficacité (plus de $75 \%$ ) et des risques de complications hémorragiques et infectieuses rares de l'expectative (Grade A). L'aspiration chirurgicale doit être préférée au curetage car elle est plus rapide, moins douloureuse et moins hémorragique (Grade B).

\section{Complications à long terme des différents traitements d'une FC du premier trimestre}

\section{Fertilité ultérieure après une FC du premier trimestre}

Le retentissement sur la fertilité ultérieure après une FC du premier trimestre a été évalué en fonction du traitement instauré (médical, chirurgical ou expectative) dans une étude ancillaire du MIST Trial (1199 femmes) [39]. Le taux de naissances vivantes cinq ans après la FC index 
était identique dans chaque groupe (79 à $82 \%$ ) (NP 2). Un suivi prospectif par téléphone a été effectué chez 261 femmes ayant participé à un essai randomisé et désireuse d'une autre grossesse [40]. Les taux de naissances vivantes dans les 4 à 9 ans suivant la randomisation étaient comparables entre les traitements chirurgical et médical (respectivement 88 et $85 \%$ ) (NP 2). Les taux de grossesses cumulées à 12 et 24 mois étaient aussi superposables (respectivement 60 et $80 \%$ ) avec un délai médian entre la randomisation et la grossesse ultérieure de huit mois dans les deux groupes [40] (NP 2).

Au total, la fertilité ultérieure est identique quel que soit le traitement d'une FC du premier trimestre (NP 2).

\section{Synéchies après traitement chirurgical d'une FC du premier trimestre}

Une méta-analyse récente a colligé dix études prospectives non randomisées comprenant un total de 912 femmes ayant bénéficié d'une hystéroscopie après les premières règles ou dans les un à douze mois suivant une FC du premier trimestre (grossesse arrêtée ou FC incomplète), quelle que soit sa prise en charge thérapeutique (médicale, chirurgicale ou expectative) [41]. Les patientes avec une histoire de FC récurrentes et sans épisode de FC de moins de 12 mois étaient exclues. Dans $86 \%$ des cas, la FC avait été traitée par chirurgie. La prévalence globale des synéchies utérines était de 19,1\% (IC $95 \%$ 12,8-27,5 \%) (NP 2). Seule une étude (47 femmes) où la FC avait été traitée médicalement ou par expectative ne montrait aucune synéchie à l'hystéroscopie. Dans les neuf autres études (781 femmes) où la FC avait été traitée par chirurgie, la prévalence des synéchies était de 18,5\% (IC $95 \%$ 12,6-26,4 \%). La sévérité des synéchies utérines, décrites chez $68 \%$ des femmes en fonction de la classification de différentes sociétés savantes internationales étaient considérées comme minimes (synéchies fines, recouvrant moins du tiers de la cavité utérine), modérées ou sévères (synéchies épaisses, recouvrant plus du tiers de la cavité utérine) dans respectivement 58, 28 et $14 \%$ des cas. Dans 
sept études (696 femmes), le risque de synéchies utérines augmentait entre celles ayant bénéficié d'un seul traitement chirurgical et celles en ayant eu au moins deux, respectivement 16 et $23 \%$, OR 2,06 (IC $95 \%$ 1,2-3,4) (NP 2). Il faut noter que le taux global d'anomalies congénitales ou acquises de la cavité utérine découvertes fortuitement lors de l'hystéroscopie parmi 675 femmes était élevé, respectivement de 12 et $29 \%$ après une FC ou après au moins deux FC ( $\mathrm{p}<0,0001)$. Les lésions d'endométrite ou la présence d'une rétention trophoblastique étaient rares, dans respectivement 6 et $3 \%$ des cas. Les auteurs de la métaanalyse n'ont pas retrouvé de données pouvant impliquer le rôle d'une inflammation ou d'une infection dans la survenue des synéchies. Les données étaient insuffisantes ou absentes concernant l'intérêt de l'administration préventive d'estrogènes ou l'influence du type de technique chirurgicale. Les auteurs n'ont pas retrouvé d'études prospectives ayant évalué le pronostic obstétrical à long terme après la découverte de synéchies utérines post-FC traitées ou pas $[41]$.

Une méta-analyse de la Cochrane Database n'avait pas retrouvé en 2010 d'essai randomisé ayant comparé les risques de synéchies utérines et de complications tardives après une aspiration chirurgicale et un simple curetage en cas de FC incomplète [38].

Au total, la découverte de synéchies utérines dans les douze mois suivant le traitement chirurgical d'une FC du premier trimestre est fréquente, environ chez une femme sur cinq (NP 2). Il s'agit le plus souvent de synéchies de sévérité minime à modérée dans $85 \%$ des cas. Le risque de synéchies utérines et leur sévérité augmente significativement au-delà de deux FC et de deux traitements chirurgicaux (NP 2). La réalisation d'une hystéroscopie diagnostique après une FC du premier trimestre n'est pas recommandée en l'absence de signes cliniques évoquant des synéchies utérines (grade C).

\section{Rétention trophoblastique prolongée}


La rétention trophoblastique prolongée après un traitement chirurgical d'une $\mathrm{FC}$ du premier trimestre est rare puisqu'elle n'est observée que dans trois pour cent des cas lorsqu'une hystéroscopie est réalisée systématiquement dans les 12 mois [41]. Il n'existe pas de définition précise et consensuelle d'une rétention trophoblastique prolongée. Dans les études ayant évalué le pronostic d'une attitude d'expectative en cas de FC incomplète, le délai avant de décider d'une évacuation chirurgicale du contenu utérin était habituellement de 6 à 8 semaines $[5,7,8$, 42]. Celle-ci est réalisée habituellement par aspiration au bloc opératoire, éventuellement sous contrôle échographique.

Aucune étude prospective n'a comparé la résection sélective par hystéroscopie à l'aspiration du contenu utérin sous contrôle échographique en cas de suspicion de rétention trophoblastique prolongée.

Deux études, rétrospectives et non randomisées, ont comparé les résultats du curetage réalisé à l'aveugle à ceux de la résection sélective par hystéroscopie $[43,44]$. Dans l'une de ces études, le taux d'échec (rétention trophoblastique persistante après la procédure chirurgicale) était de $21 \%$ dans le groupe curetage à l'aveugle $(\mathrm{n}=24)$ contre aucun dans le groupe hystéroscopie ( $\mathrm{n}=46)$ [43] (NP 4). Par contre, aucune complication opératoire ou anesthésique n'a été rapportée, quelle que soit la technique. Après hystéroscopie, le taux de grossesse ultérieure était plus élevé, bien que la différence n'était pas significative (82 versus $62 \%$ ) et le délai de conception était plus court (7 versus 11 mois, $p<0,03)$, ce qui peut s'expliquer par le nombre d'échecs plus important après un simple curetage [43] (NP 4). Dans l'autre étude $(\mathrm{n}=95)$, rétrospective également, le curetage était réalisé sous contrôle échographique [44]. Le taux de synéchies post-opératoires était significativement moins élevé après hystéroscopie qu'après curetage (4 versus $31 \%, p<0,001)$. Après hystéroscopie, le taux de grossesse ultérieure était plus élevé (69 versus $60 \%, \mathrm{p}=0,03$ ) et le délai de conception plus court (27 versus 34 mois, $\mathrm{p}=0,03)[\mathbf{4 4}](\mathrm{NP} 4)$. 
Les gels anti-adhérentiels n'ont pas été évalués dans cette indication. L'intérêt d'une hystéroscopie diagnostique de contrôle réalisée un à trois mois après n'est pas démontré.

Au total, en cas de suspicion de rétention trophoblastique prolongée après une $F C$ du premier trimestre, l'hystéroscopie diagnostique permet de confirmer le diagnostic et d'éliminer une anomalie de la cavité utérine associée (NP 2). L'hystéroscopie opératoire permet d'effectuer une résection sélective sous contrôle visuel du résidu trophoblastique (NP 4). Celle-ci semble être plus efficace que le curetage à l'aveugle, être moins responsable de synéchies post-opératoires et permettre des délais de conception plus courts (NP 4). En cas de suspicion de rétention trophoblastique prolongée, une hystéroscopie diagnostique est recommandée (Grade B).

\section{Evacuation utérine en cas de MFIU}

En cas de MFIU, le travail se met spontanément en place dans les trois semaines suivant le diagnostic pour plus de $85 \%$ des femmes [45]. Dans $10 \%$ des cas, un processus de coagulation intravasculaire disséminée lié à la mort fœtale apparait dans les quatre semaines suivant la constatation de la mort foetale et peut favoriser la survenue de complications hémorragiques au moment de l'accouchement [45].

L'analyse de la littérature est difficile en raison de la méthodologie hétérogène et de la faible puissance des études. Les critères d'inclusion (âge gestationnel, inclusion d'une majorité de grossesses encore évolutives) et les procédures médicales d'induction du travail (molécules, posologies, intervalles de prise et voies d'administration) sont très variables d'un essai à l'autre, rendant difficile la méta-analyse des résultats. Au deuxième trimestre de la grossesse, deux périodes d'inclusion sont le plus souvent distinguées par les auteurs : une précoce, généralement comprise entre 14 SA et 20-24 SA, au cours de laquelle les données sont les plus nombreuses et une période tardive comprise entre 24 et 28 SA où les données sont plus rares. 
Celles-ci sont également peu nombreuses au troisième trimestre. Les causes de la MFIU ne sont pas décrites Les utérus cicatriciels sont le plus souvent exclus.

\section{Méthodes d'évacuation utérine aux deuxième et troisième trimestres}

\section{Techniques chirurgicales d'évacuation utérine au-delà de 14 SA}

Les techniques chirurgicales sont pratiquées dans certains pays au deuxième trimestre de la grossesse, en général jusqu'à 20 ou 24 SA. Plusieurs études comparatives randomisées ou non d'origine anglo-saxonne ont montré que la chirurgie était plus efficace (obtention de la vacuité utérine) et n'augmentait pas les risques de complications opératoires et hémorragiques par rapport aux méthodes médicales d'induction du travail par le misoprostol $[\mathbf{4 6}, \mathbf{4 7}]$ (NP 3). Les complications à long terme sur les grossesses ultérieures (prématurité, perte de la grossesse) ne semblent pas augmentées après une interruption chirurgicale de la grossesse au deuxième trimestre [48, 49] (NP 4). En cas d'induction médicale du travail entre 13 et $24 \mathrm{SA}$, la principale complication est la rétention placentaire justifiant un acte chirurgical ou une délivrance manuelle complémentaires dans environ $20 \%$ des cas [46] (NP 2).

Concernant la césarienne par voie vaginale, il n'existe pas d'étude ayant comparé ses résultats à ceux d'une induction médicale du travail en cas de MFIU.

\section{Techniques médicales d'induction du travail au-delà de 14 SA}

L'efficacité de la procédure médicale est le plus souvent jugée par le délai entre le début de l'induction et l'expulsion fœtale et le taux d'expulsion dans les 24 heures suivant le début de l'induction.

Une méta-analyse de la Cochrane Database publiée en 2010 a retenu 38 études randomisées contrôlées (3490 femmes) comparant les bénéfices et les inconvénients du misoprostol administré par voie orale, vaginale ou sublinguale au placebo et à d'autres méthodes 
d'induction du travail en cas de mort fœtale ou d'anomalie fœetale justifiant une interruption de la grossesse aux deuxième et troisième trimestres [50]. La majorité des études ont été réalisées au deuxième trimestre. Le misoprostol était administré selon des protocoles très variables et comparé à des médicaments différents : géméprost (quatre études, 315 femmes), dinoprostone (six études, 410 femmes), prostaglandine F2 alpha (six études, 534 femmes), ocytocine (une étude, 76 femmes), laminaires (une étude, 68 femmes). Neuf essais n'ont inclus que des morts fœtales et 24 essais incluaient aussi bien des grossesses encore évolutives que des morts fotales. Il n'y a pas eu d'analyse distincte en fonction de la vitalité fœtale. Dans la majorité de ces études, le misoprostol n'était pas associé à la mifépristone. Le misoprostol administré par voie vaginale était aussi efficace que les autres méthodes médicamenteuses, avec des délais induction-expulsion, des taux d'expulsion dans les 24 heures et de recours à une évacuation chirurgicale complémentaire comparables. Il présentait également moins d'effets secondaires, notamment digestifs [50] (NP 2).

Les conclusions du groupe de travail du CNGOF sur l'usage du misoprostol hors AMM par rapport aux autres méthodes médicamenteuses étaient les suivantes. Les prostaglandines F2 intra-amniotiques n'ont actuellement plus leur place dans l'induction du travail [51] (NP 2). Les autres analogues des prostaglandines $\mathrm{E} 1$, la sulprostone et le géméprost, possèdent l'AMM dans l'évacuation du contenu utérin au deuxième trimestre en cas de MFIU ou d'interruption médicale de grossesse. Leur utilisation en première intention est toutefois limitée par leurs effets secondaires et leurs contre-indications (pour le sulprostone), une efficacité inférieure à celle du misoprostol et des difficultés d'utilisation et de conservation (pour le géméprost) [51] (NP 3). Au deuxième trimestre, le misoprostol est au moins aussi efficace et plutôt mieux toléré que la dinoprostone [51] (NP2). Au troisième trimestre, il y a peu d'études. Les conditions sont le plus souvent semblables au déclenchement du travail sur col non favorable et sur fœtus viable mais peuvent autoriser des doses supérieures de 
prostaglandines compte tenu de l'absence de contraintes liées à la protection du fœetus à naître [52]. A terme ou à proximité du terme, l'ocytocine et les prostaglandines E2 restent les produits ayant une AMM. Le misoprostol est une alternative selon les scores de Bishop et aux posologies du déclenchement [52].

\section{Modalités d'administration du misoprostol en cas de MFIU}

Les conclusions du groupe de travail du CNGOF sur les modalités d'administration du misoprostol hors AMM en cas d'induction médicale du travail au-delà du premier trimestre étaient les suivantes [52]. L'administration par voie vaginale réduit le délai inductionexpulsion et le taux de patientes n'ayant pas accouché dans les 24 premières heures du déclenchement par rapport à la voie orale sans augmentation des effets secondaires (NP1). La voie sublinguale semble intéressante (raccourcissement du délai induction-expulsion et augmentation du nombre de patientes ayant accouché dans les 24 premières heures par rapport à la voie vaginale) mais les données sont peu nombreuses [53] (NP2). Le délai inductionexpulsion et les doses de misoprostol nécessaires pour déclencher le travail sont diminués lorsqu'il est associé à la prise de mifépristone 24-48 heures avant le début du déclenchement [51] (NP 2). En l'absence d'utérus cicatriciel, l'utilisation du misoprostol à des doses modérées et répétées (800 à $2400 \mu \mathrm{g}$ par jour) en prises espacées de 3 à 6 heures semble présenter le meilleur compromis efficacité-tolérance mais il n'est pas possible de recommander un schéma d'administration précis [53] (NP 2).

La plupart des essais randomisés contrôlés ayant évalué l'interruption médicale de la grossesse au-delà du premier trimestre ont inclus des fœetus vivants au début de l'induction du travail et peu ont inclus des MFIU exclusivement. Comme les données de niveau de preuve élevé sont rares, notre analyse de la littérature a tenu compte des résultats d'études prospectives non 
randomisées bien menées. La méthodologie et les résultats des principales d'entre elles sont résumés dans le tableau 3 [54-65].

En 2009, une méta-analyse a colligé 14 essais randomisés contrôlés ayant comparé l'efficacité et la tolérance de différents protocoles d'administration du misoprostol pour induire le travail en cas de mort fœtale aux deuxième et troisième trimestres [66]. Différentes modalités d'administration du misoprostol ont été comparées entre elles (voies vaginale, orale ou sublinguale, doses de 100 à $400 \mu$ g toutes les 2 à 4 heures ou de 600 à $800 \mu g$ toutes les 6 à 12 heures) mais aussi à d'autres techniques d'évacuation utérine (autres prostaglandines administrées par voie vaginale ou extra-amniotique, oxytocine seule ou associée, sonde de Foley ou laminaires). Le critère de jugement principal était l'évacuation utérine complète à 48 heures. Les auteurs ont observé une grande hétérogénéité dans la méthodologie des études limitant les niveaux de preuve des résultats. Les âges gestationnels des patientes incluses étaient compris entre 13 et $40 \mathrm{SA}$ avec une majorité d'inclusions entre 14 et $24 \mathrm{SA}$. Aucune des études sélectionnées n'utilisait la mifépristone et n’a comparé le misoprostol au placebo. Les prostaglandines comparées au misoprostol étaient le géméprost $1 \mathrm{mg}$, la PGE2 et les PGF2a (quatre études). Les utérus cicatriciels étaient exclus. Certaines études incluaient des fœtus encore vivants (moins de $50 \%$ des inclusions). Le contexte des morts fotales n'était pas connu. Les résultats de cette méta-analyse sont les suivants [66].

L'administration du misoprostol par voie vaginale a été comparée à la voie orale dans quatre essais (229 femmes) selon des protocoles différents (100 à $200 \mu \mathrm{g}$ toutes les 4 à 6 heures) [5659]. Le taux d'expulsion complète à 48 heures était comparable dans les deux groupes (100\%). En cas d'administration vaginale, le délai induction-expulsion médian était significativement plus court de 2 heures (IC $95 \%-0,7$ à $-3,2$ heures, p = 0,02) (NP 1). Les effets secondaires digestifs et fébriles étaient aussi significativement moins fréquents (NP 1). L'administration vaginale était également plus efficace que la voie sublinguale (un essai entre 13 et 20 SA, 44 
femmes, 100 à $200 \mu \mathrm{g}$ toutes les 2 à 4 heures) sur le délai induction-expulsion (-5,3 heures, IC $95 \%-0,8$ à $-9,8$ heures, $\mathrm{p}=0,02$ ) avec une tendance non significative à moins d'effets indésirables mais le taux d'expulsion complète était identique entre ces deux voies [59] (NP 2). Il n'existait pas de différence sur les taux d'évacuations à 24 et 48 heures entre les doses de 400 et $600 \mu \mathrm{g}$ administrées par voie vaginale toutes les 12 heures et sur la survenue d'effets indésirables (une étude, 100 femmes) [60] (NP 2). Par contre, le délai d'expulsion médian était plus court de 3 heures avec $600 \mu \mathrm{g}$ (95\% -0,2 à -5,8 heures) (NP 2). Le misoprostol par voie vaginale était aussi efficace (taux d'expulsion à 24 heures) et aussi bien toléré que l'administration vaginale de PGE2, la dinoprostone 6 à $20 \mathrm{mg}$ toutes les 3 à 6 heures, (deux études, 135 femmes) $[\mathbf{6 7}, \mathbf{6 8}]$ et que le géméprost $1 \mathrm{mg}$ toutes les trois heures (une étude, 50 femmes) [54] (NP 2). Un essai de 120 femmes a comparé entre 18 et 40 SA le misoprostol vaginal $(50 \mu \mathrm{g}$ toutes les 6 heures) à une perfusion continue d'oxytocine [55]. Les résultats étaient stratifiés selon le score de Bishop et la rupture des membranes. Le taux d'expulsion à 48 heures était comparable entre les deux groupes mais le délai induction-expulsion moyen était plus court de 11 heures (IC $95 \%$-12 à -10 heures, 12 versus 23 heures, $p=0,004$ ) dans le groupe misoprostol (NP 2). Lorsque le score de Bishop était supérieur à 6 ou lorsque les membranes étaient rompues, il n'existait plus de différence entre les deux groupes. Un autre essai (49 femmes) a comparé entre 13 et 29 SA le misoprostol seul (200 $\mu \mathrm{g}$ toutes les 12 heures) à son association avec une perfusion d'oxytocine $(1,5 \mathrm{mU} / \mathrm{min})$ débutée lors de l'administration de la première dose de misoprostol [69]. Cette association était plus efficace en termes d'expulsion à 24 heures (RR 0,45 IC $95 \%$ 0,24-0,85) et de délai induction-expulsion moyen (-11,5 heures IC -4 à -19 heures, 16 versus 28 heures) (NP 2). Mais les doses de misoprostol administrées dans cette étude étant faibles (200 $\mu \mathrm{g}$ toutes les 12 heures), il est difficile d'affirmer la supériorité d'une perfusion d'oxytocine associée à la prise de misoprostol. Enfin, un autre essai de 68 femmes n'observait pas de différence entre 12 et 22 
SA sur les taux d'expulsion à 24 et 48 heures en associant des laminaires à l'administration de misoprostol vaginal (200 $\mu$ g toutes les 12 heures) [70] (NP 2). Au final, les auteurs de cette méta-analyse concluaient que leurs résultats devaient être interprétés avec précaution en raison de la faible puissance et de la grande hétérogénéité de la méthodologie des études sélectionnées et compte tenu qu'elles étaient principalement réalisées entre 13 et 28 SA [66]. D’autres études plus puissantes et plus homogènes sont donc nécessaires pour déterminer le meilleur protocole médicamenteux en cas de MFIU au-delà de 14 SA.

L'analyse des résultats des études prospectives portant sur l'utilisation seule du misoprostol (sans mifépristone associée) dans l'induction du travail en cas de MFIU au-delà de 14 SA donne les résultats suivants (tableau 3). Des doses élevées de misoprostol, supérieures à 400 $\mu \mathrm{g}$ par prise, ne semblaient pas apporter plus de bénéfices en termes de succès et de rapidité d'expulsion que des doses de 200 à $400 \mu$ g (NP 3). Par contre, des intervalles de prises courts de 4 à 6 heures semblaient être plus efficaces que des délais plus prolongés de 12 heures (NP 3). L'administration buccale (préjugale) d'une dose d'au moins $200 \mu \mathrm{g}$ toutes les six heures mérite d'être davantage évaluée [62]. Une administration par voie vaginale de misoprostol à la dose de 200 à $400 \mu \mathrm{g}$ toutes les quatre heures permet d'obtenir des délais induction-expulsion moyens compris entre 10 et 14 heures et des taux d'expulsion complète à 24 heures compris entre 84 et $100 \%$ (tableau 3) (NP 2). Une perfusion d'oxytocine et le recours à un geste chirurgical complémentaire étaient respectivement nécessaires dans 11 à $46 \%$ et 6 à $40 \%$ des cas en fonction de l'âge gestationnel et des modalités d'administration du misoprostol [57, 61] (NP 3).

Au total, le protocole d'administration du misoprostol le plus efficace (délais inductionexpulsion et taux d'expulsion dans les 24 heures) en cas de MFIU au-delà de 14 SA et en l'absence d'utérus cicatriciel semble être la dose de 200 à $400 \mu \mathrm{g}$ par voie vaginale, renouvelée toutes les 4 à 6 heures (Grade B). 
Intérêt d'une administration préalable de mifépristone avant l'induction du travail par le misoprostol en cas de MFIU au-delà de 14 SA

Il n'existe pas d'essai randomisé contrôlé ayant évalué dans cette situation clinique les bénéfices de la mifépristone par rapport à l'utilisation seule du misoprostol.

Une étude prospective non randomisée indienne ayant inclus 40 MFIU après 28 SA observait une diminution significative du délai induction-expulsion moyen après une prise orale de 200 mg de mifépristone 36 heures avant une dose orale de 50 à $100 \mu \mathrm{g}$ (respectivement avant et après $37 \mathrm{SA}$ ) de misoprostol toutes les trois heures (quatre doses maximum) par rapport à une prise isolée de la même dose de misoprostol (6,7 versus 11,8 heures, p < 0,05) [65] (NP 3). La dose cumulée totale de misoprostol était également diminuée en cas de prise préalable de mifépristone $(1,6$ versus $3, \mathrm{p}<0,01)$. Il faut noter que dans cette série, $60 \%$ des patientes ont expulsé dans les 36 heures suivantes la prise de mifépristone. La parité, l'âge gestationnel et le score de Bishop initial n'affectaient pas les taux de succès dans les deux groupes [65].

Au total, en cas de MFIU, l'administration préalable d'une dose de 200 mg de mifépristone au moins 24 heures avant celle de misoprostol améliore les délais d'expulsion et diminue les besoins en misoprostol (NP 3). Elle est donc recommandée (Grade C).

Intérêt d'une préparation cervicale préalable par la pose de laminaires ou d'une sonde de dilatation

Les bénéfices d'une préparation cervicale par la pose de laminaires 12 à 24 heures avant le début de l'induction du travail par le misoprostol sont controversés en raison de l'absence d'étude randomisée de puissance suffisante. Six essais randomisés ayant évalué l'intérêt d'une préparation cervicale par des laminaires ou à l'aide d'une sonde de Foley avant une évacuation chirurgicale du contenu utérin au deuxième trimestre de la grossesse, entre 14 et 24 ont été 
colligés dans une revue de la Cochrane Database [71]. Aucune méta-analyse n'a pu être effectuée en raison de la trop grande hétérogénéité des méthodologies. Les dilatateurs osmotiques étaient plus efficaces que le misoprostol seul (400 $\mu \mathrm{g}$ par voie vaginale) pour faciliter la dilatation cervicale et réduire le temps opératoire. Par contre, il n'existait pas de différence concernant les risques de complications opératoires [71] (NP 2).

Au total, les données sont insuffisantes pour dire si les laminaires sont bénéfiques lors d'une induction du travail en cas de MFIU.

\section{Prise en charge de l'accouchement}

Il n'existe pas d'études randomisées ayant comparé les différentes alternatives de prise en charge de l'accouchement après une induction médicale du travail pour MFIU au-delà du premier trimestre (poursuite des doses de misoprostol jusqu'à l'expulsion, direction du travail par une perfusion d'oxytocine lorsqu'une dose maximale de misoprostol a été atteinte ou dès que le col s'est modifié et que les contractions utérines sont régulières). Un essai randomisé a comparé chez 22 femmes présentant une MFIU les conséquences sur le déroulement du travail de l'analgésie péridurale par rapport à la sédation par morphine parentérale [72]. Dans le groupe analgésie péridurale, le délai d'expulsion moyen et la vitesse de dilatation cervicale au court de la première phase du travail étaient respectivement plus court $(5,4$ versus 8,7 heures, $\mathrm{p}=0,02)$ et plus rapide (3 versus $1 \mathrm{~cm}$ par heure, $\mathrm{p}=0,01)(\mathrm{NP} 2)$.

En l'absence de mise en travail, aucune étude randomisée n'a évalué les doses maximales de misoprostol et l'intérêt d'une fenêtre thérapeutique avant de reprendre l'induction médicale du travail par le misoprostol ou par une perfusion d'oxytocine.

\section{Risque de rétention placentaire après l'expulsion}


Les risques de rétention placentaire sont très variables d'une étude à l'autre en raison de la grande hétérogénéité des protocoles médicamenteux utilisés, des délais accordés entre l'expulsion fœetale et celle du placenta et des âges gestationnels. Les taux de rétention placentaires rapportés ont tendance à être inversement proportionnels à l'âge gestationnel, de 26 à $60 \%$ avant 22-24 SA et de 5 à $23 \%$ au-delà de 24-28 SA [61, 63] (NP 3). Les méthodes d'évacuation utérine varient en fonction de l'âge gestationnel entre un geste chirurgical effectué au bloc opératoire et dont les modalités techniques sont rarement décrites et une délivrance artificielle manuelle réalisée le plus souvent lorsque l'âge gestationnel est supérieur à 24 SA. Les délais de réalisation de l'évacuation utérine complémentaire après l'expulsion fœtale sont également très variables d'une étude à l'autre, en général de plus d'une heure. Entre 14 et $28 \mathrm{SA}$, la délivrance spontanée se produit dans 60 à $90 \%$ des cas dans l'heure qui suit l'expulsion fœetale $[\mathbf{6 1}, \mathbf{7 3 - 7 5}$ (NP 3). Des délais prolongés de deux à quatre heures ne semblent pas être associés à une augmentation du risque hémorragique [73, 74] (NP 3). L'administration d'utérotoniques après l'expulsion fœtale (oxytocine intramusculaire ou misoprostol oral) ne semble pas accélérer la délivrance placentaire mais les quelques essais randomisés ont certainement une puissance trop faible pour montrer une différence significative $[\mathbf{7 3}, \mathbf{7 5}](\mathrm{NP} 3)$.

Au total, le risque de rétention placentaire semble être inversement proportionnel à l'âge gestationnel (NP 3). Le risque hémorragique ne semble pas être majoré avec l'augmentation du délai expulsion foetale-délivrance (NP 3). Les données actuelles ne permettent pas de définir un délai au-delà duquel il faut effectuer systématiquement une évacuation utérine complémentaire après l'expulsion foetale. Il n'existe pas de preuve suffisante pour dire que la délivrance dirigée par administration d'ocytociques est utile après une induction du travail au deuxième trimestre. 


\section{Situations cliniques particulières}

\section{Particularité de l'induction du travail en cas de MFIU à proximité du terme de la grossesse}

La majorité des données sont disponibles entre 14 et 28 SA et il n'existe pas d'études randomisées ayant comparé l'efficacité et l'innocuité des différentes techniques médicales audelà de 28 SA (modalités d'administration du misoprostol, autres prostaglandines, oxytocine, laminaires, ballonnet de dilatation cervicale).

\section{Utérus unicicatriciel et multicicatriciel}

En 2012, les Recommandations de Pratique Clinique du CNGOF avaient conclu qu'une tentative de voie basse était préférable à une césarienne programmée en cas de MFIU associée à un utérus cicatriciel (accord professionnel) [76].

Quelques essais prospectifs de faible puissance (moins de 100 femmes incluses) ont évalué l'usage du misoprostol chez des femmes ayant au moins un antécédent de césarienne en cas de MFIU [51, 53]. Les protocoles médicamenteux et l'utilisation des ocytociques étaient très variables d'une étude à l'autre. Les taux de succès et les délais induction-expulsion étaient comparables entre les groupes de patientes présentant un utérus sain et ceux de patientes présentant un utérus cicatriciel, même lorsqu'une demi-dose de misoprostol était utilisée (NP 3). Les taux d'hémorragies et de transfusion étaient également comparables. L'augmentation du risque de rupture utérine en cas d'utérus cicatriciel n'était pas retrouvée dans toutes les études, probablement en raison de la faiblesse des effectifs et de la rareté de cet évènement [53] (NP 3) Les ruptures utérines rapportées étaient survenues soit lors de l'administration de doses moyennes à élevées de misoprostol (à partir de $200 \mu \mathrm{g}$ toutes les trois heures), soit en cas d'administration successive de misoprostol et d'ocytocine [53] (NP 3). Les situations à risque de rupture utérine semblent être une dose cumulée élevée de misoprostol (plus de $2400 \mu \mathrm{g}$ par 
24 heures) et/ou l'association de plusieurs utérotoniques (NP 3). Le risque de rupture utérine en cas d'utérus cicatriciel justifie l'utilisation des doses minimales efficaces [53] (NP3). Il semble encore plus important chez ces patientes d'optimiser la préparation cervicale par l'administration de mifépristone 36 à 48 heures avant le début du déclenchement et éventuellement l'utilisation de laminaires afin de permettre un accès plus rapide à la poche des eaux. Il n'est pas démontré que l'augmentation de la dose de mifépristone à 600 mg soit utile dans cette situation [51].

Les autres techniques de maturation cervicale autres que médicamenteuses, telles que le ballonnet transcervical, n'ont pas été évaluées dans cette indication chez les patientes ayant un antécédent de césarienne.

Au total, il n'existe pas de donnée pour recommander une césarienne en cas de MFIU chez une patiente ayant un ou plusieurs antécédents de césarienne. Le risque de rupture utérine semble être augmenté en cas d'induction médicale du travail par le misoprostol (NP 4). Les doses minimales efficaces doivent être utilisées (100 à $200 \mu \mathrm{g}$ toutes les 4 à 6 heures) (Grade C). Il semble encore plus important chez ces patientes d'optimiser la préparation cervicale par l'administration de mifépristone, 200 à $600 \mathrm{mg} 36$ à 48 heures avant le début du déclenchement, et éventuellement par l'utilisation de laminaires. Il est recommandé de respecter un délai d'au moins trois heures entre l'administration des prostaglandines et d'autres utérotoniques tels que l'ocytocine (Grade C).

Pathologies placentaires : anomalies d'insertion placentaire, hématome rétro-placentaire Il n'existe pas de donnée de niveau de preuve élevé sur les modalités d'accouchement en cas de MFIU associée à la présence d'un placenta prævia ou à une suspicion de placenta accreta. 
Rétention placentaire prolongée après une évacuation utérine aux deuxième et troisième trimestres

En 2013, une méta-analyse de la Cochrane Database n'avait pas retrouvé d'étude randomisée contrôlées ayant évalué les différentes méthodes thérapeutiques en cas de FCS incomplète entre 13 et 24 SA [37]. 


\section{Références bibliographiques}

1. NICE Clinical Guideline. Ectopic pregnancy and miscarriage: Diagnosis and initial management in early pregnancy of ectopic pregnancy and miscarriage. National Collaborating Centre for Women's and Children's Health. London: RCOG; 2012.

2. Graziosi GCM, Mol BW, Ankum WM, Bruinse HW. Management of early pregnancy loss. Int J Gynecol Obstet 2004; 86: 337-46.

3. Neilson JP, Hickey M, Vazquez J. Medical treatment for early fetal death (less than 24 weeks). Cochrane Database Syst Rev 2006; Issue 3: CD002253.

4. Sotiriadis A, Makrydimas G, Papatheodorou S, Ioannidis JPA. Expectant, medical or surgical management of first-trimester miscarriage: a meta-analysis. Obstet Gynecol 2005; 105: 1104-13.

5. Trinder J, Brocklehurst P, Porter R, Read M, Vyas S, Smith L. Management of miscarriage: expectant, medical or surgical? Results of randomised controlled trial (miscarriage treatment (MIST) trial). BMJ 2006; 332: 1235-40.

6. Zhang J, Gilles JM, Barnhart K, Creinin MD, Westhoff C, Frederick MM. A comparison of medical management with misoprostol and surgical management for early pregnancy failure. N England J Med 2005; 353: 761-9.

7. Nanda K, Lopez LM, Grimes DA, Peloggia A, Nanda G. Expectant care versus surgical treatment for miscarriage. Cochrane Database Syst Rev 2012; Issue 3: CD003518.

8. Luise C, Jermy K, May C, Costello G, Collins WP, Bourne TH. Outcome of expectant management of spontaneous first trimester miscarriage: obsvervational study. BMJ 2002; 324: $873-5$.

9. Torre A, Huchon C, Bussieres L, Machevin E, Camus E, Fauconnier A. Immediate versus delayed medical treatment for first-trimester miscarriage : a randomized trial. Am J Obstet Gynecol 2013; 206:215.e1-6. 
10. May W, Gülmezoglu AM, Ba-Thike K. Antibiotics for incomplete abortion. Cochrane Database Syst Rev 2007; 17:CD001779.

11. Titapant V, Cherdchoogieat P. Effectiveness of cefoxitin on preventing endometritis after uterine curettage for spontaneous incomplete abortion: a randomized controlled trial study. J Med Assoc Thai. 2012; 95 :1372-7.

12. Beucher G, Dolley P, Carles G, Salaun F, Asselin I, Dreyfus M. Misoprostol : utilisation hors AMM au premier trimestre de la grossesse (fausses couches spontanées, interruptions médicales et volontaires de grossesse). J Gynecol Obstet Biol Reprod 2014; $43: 123-45$.

13. Lister MS, Shaffer LE, Bell JG, Lutter KQ, Moorma KH. Randomized, double-blind, placebo-controlled trial of vaginal misoprostol of early pregnancy failures. Am L Obstet Gynecol 2005; 193:1338-43.

14. Tang OS, Lau WNT, Ng EHY, Lee SWH, Ho PC. A prospective randomized study to compare the use of repeated doses of vaginal with sublingual misoprostol in the management of first trimester silent miscarriage. Human Reprod 2003; 18:176-81.

15. Tang OS, Ong CY, Tse KY, Ng EH, Lee SW, Ho PC. A randomized trial to compare the use of sublingual misoprostol with or without an additional 1 week course for the management of first trimester silent miscarriage. Hum Reprod 2006;21:189-92.

16. Odeh M, Tendler R, Kais M, Maximovsky O, Ophir E, Bornstein J. Early pregnancy failure: factors affecting successful medical treatment. Isr Med Assoc J 2010; 12: 325-8.

17. Creinin MD, Huang X, Westhoff C, Barnhart K, Gilles JM, Zhang J. Factors related to successful misoprostol treatment for early pregnancy failure. Obstet Gynecol 2006; 107: 901-7. 
18. Robledo C, Zhang J, Troendle J, Barnhart K, Creinin MD, Westhoff C, et al. Clinical indicators for success of misoprostol treatment after early pregnancy failure. Int $\mathbf{J}$ Gynaecol Obstet 2007; 99: 46-51.

19. Reynolds A, Ayres-de-Campos D, Costa MA, Montenegro N. How should success be defined when attempting medical resolution of first-trimester missed abortion? Eur $\mathbf{J}$ Obstet Gynecol Reprod Biol 2005; 118: 71-6.

20. Gronlund A, Grønlund L, Clevin L, Andersen B, Palmgren N, Lidegaard Ø. Management of missed abortion: comparison of medical treatment with either mifepristone + misoprostol or misoprostol alone with surgical evacuation. A multicenter trial in Copenhagen county, Denmark. Acta Obstet Gynecol Scand 2002; 81: 1060-5.

21. Stockheim D, Machtinger R, Wiser A, Dulitzky M, Soriano D, Goldenberg M et al. A randomized prospective study of misoprostol or mifepristone followed by misoprostol when needed for the treatment of women with early pregnancy failure. Fertil Steril 2006; 86: $956-60$.

22. Kollitz KM, Meyn LA, Lohr PA, Creinin MD. Mifepristone and misoprostol for early pregnancy failure: a cohort analysis. Am J Obstet Gynecol 2011; 204: 386 e1-6.

23. Davis AR, Hendlish SK, Westhoff C, Frederick MM, Zhang J, Gilles JM, et al. Bleeding patterns after misoprostol vs surgical treatement of early pregnancy failure: results from a randomized trial. Am J Obstet Gynecol 2007; 196:31.e1-7.

24. Chen BA, Reeves MF, Creinin MD, Gilles JM, Barnhart K, Westhoff CW, et al. Misoprostol for treatment of early pregnancy failure in women with previous uterine surgery. Am J Obstet Gynecol 2008; 198: 626 e1-5.

25. Kong GW, Lok IH, Yiu AK, Hui AS, Lai BP, Chung TK. Clinical and psychological impact after surgical, medical or expectant management of first-trimester miscarriage--a randomised controlled trial. Aust N Z J Obstet Gynaecol 2013;53: 170-7. 
26. Rausch M, Lorch S, Chung K, Frederick M, Zhang J, Barnhart K. A cost-effectiveness analysis of surgical versus medical management of early pregnancy loss. Fertil Steril 2012; 97:355-60.

27. Graziosi GC, van der Steeg JW, Reuwer PH, Drogtrop AP, Bruinse HW, Mol BW. Economic evaluation of misoprostol in the treatment of early pregnancy failure compared to curettage after an expectant management. Hum Reprod 2005, 20:1067-71.

28. Kauff ND, Chelmow D, Kawada CY. Intractable bleeding managed with Foley catheter tamponade after dilation and evacuation. Am J Obstet Gynecol 1995; 173:957-8.

29. Olamijulo J, Doufekas K. Intrauterine balloon tamponade for uncontrollable bleeding during first trimester surgical termination of pregnancy. J Obstet Gynaecol 2007;27:440-1

30. Madden T, Burke A. Successful management of second-trimester postabortion hemorrhage with an intrauterine tamponade balloon. Obstet Gynecol 2009;113:501-3.

31. Borgatta L, Chen AY, Reid SK, Stubblefield PG, Christensen DD, Rashbaum WK. Pelvic embolization for treatment of hemorrhage related to spontaneous and induced abortion. Am J Obstet Gynecol 2001; 185:530-6.

32. Haddad L, Delli-Bovi L. Uterine artery embolization to treat hemorrhage following second-trimester abortion by dilatation and surgical evacuation. Contraception 2009; $79: 452-5$.

33. Steinauer JE, Diedrich JT, Wilson MW, Darney PD, Vargas JE, Drey EA. Uterine artery embolization in postabortion hemorrhage. Obstet Gynecol 2008;111:881-9.

34. Casikar I, Bignardi T, Riemke J, Alhamdan D, Condous G. Expectant management of spontaneous first trimester miscarriage: prospective validation of the two week rule. Ultrasound Obstet Gynecol 2010; 35:223-7. 
35. Casikar I, Lu C, Oates J, Bignardi T, Alhamdan D, Condous G. The use of power Doppler colour scoring to predict successful expectant management in women with an incomplete miscarriage. Hum Reprod 2012; 27:669-75.

36. Verschoor MA, Lemmers M, Bossuyt PM, Graziosi GC, Hajenius PJ, Hendriks DJ et al. Surgical versus expectant management in women with an incomplete evacuation of the uterus after treatment with misoprostol for miscarriage: the MisoREST trial. BMC Pregnancy Childbirth. 2013; 13:102.

37. Neilson JP, Gyte GM, Hickey M, Vazquez, Dou L. Medical treatments for incomplete miscarriage (less than 24 weeks). Cochrane Database Syst Rev 2013; Issue 3: CD007223.

38. Tunçalp O, Gulmezoglu AM, Souza JP. Surgical procedures to evacuating incomplete abortion. Cochrane Database Syst Rev 2010; Issue 9: CD001993.

39. Smith LFP, Ewings PD, Quinlan C. Incidence of pregnancy after expectant, medical, or surgical management of spontaneous first trimester miscarriage: long term follow-up of miscarriage treatment (MIST) randomised controlled trial. BMJ 2009; 339: b3827.

40. Tam WH, Tsui MH, Lok IH, Yip SK, Yuen PM, Chung TK. Long-term reproductive outcome subsequent to medical versus surgical treatment for miscarriage. Hum Reprod $2005 ; 20: 3355-9$.

41. Hooker AB, Lemmers M, Thurkow AL, Heymans MW, Opmeer BC, Brölmann HA, et al. Systematic review and meta-analysis of intrauterine adhesions after miscarriage: prevalence, risk factors and long-term reproductive outcome. Hum Reprod Update 2014 ; $20: 262-78$.

42. Van den Bosch T, Daemen A, Van Schoubroeck D, Pochet N, De Moor B, Timmerman D. Occurrence and outcome of residual trophoblastic tissue: a prospective study. J Ultrasound Med 2008; 27:357-61. 
43. Cohen SB, Kalter-Ferber A, Weisz BS, Zadel Y, Seidman DS, Maschiach S et al. Hysteroscopy may be the method of choice for management of residual trophoblastic tissue. J Am Assoc Gynecol Laparosc 2001; 8: 199-202.

44. Rein DT, Schmidt T, Hess AP, Volkmer A, Schondorf T, Breidenbach M. Hysteroscopic management of residual trophoblastic tissue is superior to ultrasound-guided curettage. $\mathbf{J}$ Minim Invasive Gynecol 2011; 18; 774-8.

45. Royal College of Obstetricians and Gynaecologists. Late intrauterine fetal death and stillbirth. RCOG guideline N55. London: RCOG Press 2010.

46. Bryant AG, Grimes DA, Garrett JM, Stuart GS. Second-trimester abortion for fetal anomalies or fetal death: labor induction compared with dilation and evacuation. Obstet Gynecol 2011 117: 788-92.

47. Kelly T, Suddes J, Howel D, Hewison J, Robson S. Comparing medical versus surgical termination of pregnancy at 13-20 weeks of gestation: a randomised controlled trial. BJOG 2010; 117:1512-20.

48. Chasen ST, Kalish RB, Gupta M, Kaufman J, Chervenak FA. Obstetric outcomes after surgical abortion at $>$ or $=20$ weeks' gestation. Am J Obstet Gynecol. 2005;193:1161-4.

49. Jackson JE, Grobman WA, Haney E, Casele H. Mid-trimester dilation and evacuation with laminaria does not increase the risk for severe subsequent pregnancy complications. Int J Gynaecol Obstet 2007; 96:12-5.

50. Dodd JM, Crowther CA. Misoprostol for induction of labour to terminate pregnancy in the second or third trimester for women with a fetal anomaly or after intrauterine fetal death. Cochrane Database Syst Rev 2010, Issue 4: CD004901.

51. Pluchon M, Winer N. Misoprostol dans les IMG aux $2^{\text {ème }}$ et $3^{\text {ème }}$ trimestres. Essais comparatifs. J Gynecol Obstet Biol Reprod 2014; 43 :162-8. 
52. Marret H, Simon E, Beucher G, Dreyfus M, Gaudineau A, Vayssière C et al. Etats des lieux et expertise de l'usage hors AMM du misoprostol en gynécologie obstétrique : travail du CNGOF. J Gynecol Obstet Biol Reprod 2014; 43 : 107-13.

53. Clouqueur E, Coulon C, Vasst P, Chauvet A, Deruelle P, Subtil D et al. Utilisation du misoprostol pour l'induction du travail en cas de MIU ou d'IMG au deuxième ou au troisième trimestre de la grossesse : efficacité, posologie, voie d'administration, effets secondaires, utilisation en cas d'utérus cicatriciel. J Gynecol Obstet Biol Reprod 2014; $43: 146-61$.

54. Eng NS, Guan AC. Comparative study of intravaginal misoprostol with gemeprost as an abortifacient in second trimester missed abortion. Aust N Z J Obstet Gynaecol $1997 ; 37: 331-4$

55. Nakintu N. A comparative study of vaginal misoprostol and intravenous oxytocin for induction of labour in women with intra uterine fetal death in Mulago Hospital, Uganda. Afr Health Sci 2001; 1:55-9.

56. Chittacharoen A, Herabutya Y, Punyavachira P. A randomized trial of oral and vaginal misoprostol to manage delivery in cases of fetal death. Obstet Gynecol 2003;101:70-3.

57. Fadalla FA, Mirghani OA, Adam I. Oral misoprostol vs. vaginal misoprostol for termination of pregnancy with intrauterine fetal demise in the second-trimester. Int $\mathbf{J}$ Gynecol Obstet 2004; 86:52-3.

58. Nyende L, Towobola OA, Mabina MH. Comparison of vaginal and oral misoprostol, for the induction of labour in women with intrauterine fetal death. East Afri Med J 2004; $81: 179-82$.

59. Caliskan E, Dilbaz S, Doger E, Ozeren S, Dilbaz B. Randomized comparison of 3 misoprostol protocols for abortion induction at 13-20 weeks of gestation. J Reprod Med 2005; 50:173-80. 
60. Niromanesh S, Hashemi-Fesharaki M, Mosavi-Jarrahi A. Second trimester abortion using intravaginal misoprostol. Int J Gynaecol Obstet 2005; 89:276-7.

61. Van Mensel K, Claerhout F, Debois P, Keirse MJ, Hanssens M. A randomized controlled trial of misoprostol and sulprostone to end pregnancy after fetal death. Obstet Gynecol Int 2009:496320.

62. Bracken H, Ngoc NT, Banks E, Blumenthal PD, Derman RJ, Patel A et al. Buccal misoprostol for IUFD Research Group. Buccal misoprostol for treatment of fetal death at 14-28 weeks of pregnancy: a double-blind randomized controlled trial. Contraception. 2014; 89: 187-92.

63. Wagaarachchi PT, Ashok PW, Narvekar NN, Smith NC, Templeton A. Medical management of late intrauterine death using a combination of mifepristone and misoprostol. BJOG 2002; 109:443-7.

64. Fairley TE, Mackenzie M, Owen P, Mackenzie F. Management of late intrauterine death using a combination of mifepristone and misoprostol-experience of two regimens. Eur $\mathbf{J}$ Obstet Gynecol Reprod Biol 2005; 118:28-31.

65. Sharma D, Singhal SR, Poonam, Paul A, Kunika. Comparison of mifepristone combination with misoprostol and misoprostol alone in the management of intrauterine death: condensation - misoprostol and mifepristone combination is more effective than misoprostol alone in the management of intrauterine death. Taiwan J Obstet Gynecol 2011;50:322-5.

66. Gómez Ponce de León R, Wing DA. Misoprostol for termination of pregnancy with intrauterine fetal demise in the second and third trimester of pregnancy - a systematic review. Contraception 2009; 79:259-71.

67. Jain JK, Mishell Jr DR. A comparison of intravaginal misoprostol with prostaglandin E2 for termination of second-trimester pregnancy. N Engl J Med 1994;331:290-3. 
68. Makhlouf AM, Al-Hussaini TK, Habib DM, Makarem MH. Second trimester pregnancy termination: comparison of three different methods. J Obstet Gynecol 2003; 23:407-11.

69. Hidar S, Fekih M, Chaieb A, Bibi M, Mellouli R, Khairi H. Apport de l'association d'ocytocine au misoprostol administré en intravaginal au cours des interruptions de grossesses entre 13 et 29 semaines d'aménorrhée. Essai clinique prospectif randomisé. J Gynecol Obstet Biol Reprod 2001;30:439-43.

70. Jain JJ, Mishell DR. A comparison of misoprostol with and without laminaria tents for induction of second-trimester abortion. Am J Obstet Gynecol 1996; 175:173-7.

71. Newmann SJ, Dalve-Endres A, Diedrich JT, Steinauer JE, Meckstroth K, Drey EA. Cervical preparation for second trimester dilation and evacuation. Cochrane Database Syst Rev 2010; 8:CD007310.

72. Lurie S, Blickstein I, Feinstein M, Matzkel A, Ezri T, Soroker D. Influence of epidural anaesthesia on the course of labour in patients with antepartum fetal death. Aust $\mathrm{N} \mathrm{Z} \mathrm{J}$ Obstet Gynaecol 1991; 31:227-8.

73. Leader J, Bujnovsky M, Carlan SJ, Triana T, Richichi K. Effect of oral misoprostol after second-trimester delivery: a randomized, blinded study. Obstet Gynecol 2002; 100:68994.

74. Green J, Borgatta L, Sia M, Kapp N, Saia K, Carr-Ellis S et al. Intervention rates for placental removal following induction abortion with misoprostol. Contraception 2007; $76: 310-3$

75. Dickinson JE, Evans SF. A comparison of oral misoprostol with vaginal misoprostol administration in second-trimester pregnancy termination for fetal abnormality. Obstet Gynecol 2003; 101:1294-9. 
76. Deruelle P, Lepage J, Depret S, Clouqueur E. Mode de déclenchement du travail et conduite du travail en cas d'utérus cicatriciel. J Gynecol Obstet Biol Reprod 2012;41:788802. 

Tableau 1: Comparaison des bénéfices et risques du traitement médical par le misoprostol et de l'évacuation chirurgicale en cas de grossesse arrêtée au premier trimestre. Résultats des méta-analyses et des principales études randomisées. Benefitsrisks balance between medical treatment using misoprostol and surgical uterine evacuation in case of early miscarriage. Main randomized studies and meta-analysis results.

\begin{tabular}{|c|c|c|c|}
\hline & Misoprostol & $\begin{array}{l}\text { Evacuation } \\
\text { chirurgicale }\end{array}$ & $\begin{array}{l}\text { OR ou RR (IC } 95 \\
\%)\end{array}$ \\
\hline \multicolumn{4}{|c|}{ Recours à un traitement chirurgical non programmé (\%) } \\
\hline NICE $2012^{a}[\mathbf{1}], n=4739$ & 20 & 3 & $\operatorname{RR} 8,13(6,26-10,55)$ \\
\hline Taux d'expulsion complète $(\%)$ & \multicolumn{3}{|c|}{ Sans recours à un traitement chirurgical non programmé } \\
\hline Neilson $2006^{\mathbf{b}}[\mathbf{3}], \mathrm{n}=254$ & 60 & 97 & RR $0,42(0,34-0,52)$ \\
\hline Graziosi $2004^{\mathrm{c}}[2], \mathrm{n}=401$ & 83 & 98 & OR $0,05(0,03-0,09)$ \\
\hline Zhang $2005^{\mathrm{d}}[\mathbf{6}], \mathrm{n}=652$ & 84 & 97 & I \\
\hline Trinder $2006^{\mathrm{e}}[\mathbf{5}], \mathrm{n}=801$ & 77 & 94 & I \\
\hline Traumatismes opératoires (\%) & \multicolumn{3}{|c|}{ Perforations utérines, déchirures cervicales } \\
\hline Neilson 2006 [3], $\mathrm{n}=154$ & 0 & 1,3 & NS \\
\hline Graziosi 2004 [2], n = 419 & 0 & 1,9 & NS \\
\hline Hémorragies sévères (\%) & \multicolumn{3}{|c|}{ transfusion sanguine } \\
\hline NICE $2012[\mathbf{1}], \mathrm{n}=2416$ & 1,1 & 0,8 & NS \\
\hline Graziosi 2004 [2], n = 845 & 0,2 & 0,2 & NS \\
\hline Infections (\%) & \multicolumn{3}{|c|}{ Nécessitant une antibiothérapie } \\
\hline NICE $2012[\mathbf{1}], \mathrm{n}=2568$ & 1,6 & 2,2 & NS \\
\hline Graziosi 2004 [2], n = 765 & 2,6 & 3,7 & NS \\
\hline Zhang 2005 [6], n = 652 & 0,4 & 0 & NS \\
\hline Trinder 2006 [5], $\mathrm{n}=801$ & 3 & 4 & NS \\
\hline Troubles digestifs secondaires $(\%)$ & Nausées, vomiss & ments, diarrhées & \\
\hline
\end{tabular}


NICE 2012 [1], n = 7704

\section{Consultations non programmées (\%)}

NICE 2012 [1], $\mathrm{n}=2401$

\section{Hospitalisations non programmées (\%)}

OR : Odds ratio ; $\mathbf{R R}$ : risque relatif ; IC : intervalle de confiance ; NS : non significatif.

* Recours à un traitement chirurgical non programmé

a : Méta-analyse de 18 essais (4739 femmes) ayant comparé le misoprostol par voie vaginale (doses variables) à l'aspiration chirurgicale.

b : Méta-analyse de trois études randomisées (254 femmes) ayant comparé le misoprostol par voie vaginale (800 $\mu \mathrm{g}$ éventuellement renouvelés) à l'aspiration chirurgicale, délai avant décision d'échec du traitement médical variable compris entre 10 heures et une semaine.

c : Méta-analyse de cinq études randomisées (401 femmes) ayant comparé le misoprostol par voie vaginale (800 $\mu \mathrm{g}$ éventuellement renouvelés) ou par voie orale (200 à $400 \mu \mathrm{g}$ répétés toutes les 4 à 6 heures) à l'aspiration chirurgicale.

d : Essai randomisé multicentrique (quatre centres universitaires américains) ; grossesse arrêtée définie par un embryon sans activité cardiaque ayant une longueur cranio-caudale comprise entre 5 et $40 \mathrm{~mm}$ ou par un sac gestationnel sans image embryonnaire mesurant entre 16 et $45 \mathrm{~mm}$; groupe misoprostol : $800 \mu \mathrm{g}$ par voie vaginale, éventuellement renouvelés à 48 heures, aspiration chirurgicale à 8 jours en cas de persistance du sac gestationnel ; groupe chirurgie : aspiration électrique au bloc opératoire ou manuelle en ambulatoire en fonction du centre ; évaluation des critères de jugement (efficacité, effets adverses) jusqu'au 30 ème jour après l'inclusion (évaluation à J3, J8, J15 et J30).

e : Essai randomisé multicentrique (sept centres hospitaliers anglais) ; grossesse arrêtée définie par un embryon sans activité cardiaque ayant une longueur cranio-caudale d'au moins $6 \mathrm{~mm}$ ou par un sac gestationnel mesurant au moins $20 \mathrm{~mm}$; groupe traitement médical : mifépristone 200mg par voie orale 24-48 heures avant misoprostol $800 \mu \mathrm{g}$ par voie vaginale, aspiration chirurgicale à 8 heures en cas de persistance du sac gestationnel ; groupe chirurgie : aspiration électrique au bloc opératoire sous anesthésie générale ; évaluation des critères de jugement (efficacité, effets adverses) à $\mathrm{J} 14$ et à huit semaines de la randomisation. 
Tableau 2 : Avantages et inconvénients des méthodes thérapeutiques pour l'obtention de la vacuité utérine après une fausse couche (FC)

du premier trimestre (grossesse arrêtée et FC incomplète). Therapeutic options for obtaining of uterine vacuity after an early miscarriage.

Advantage and disadvantage of each method.

\begin{tabular}{|c|c|c|}
\hline & Avantages & Inconvénients \\
\hline Chirurgie & $\begin{array}{l}\text { - Taux de succès élevé a (95-98 \%) (NP 1) } \\
\text { - Prise en charge rapide, ambulatoire (1 jour) }\end{array}$ & $\begin{array}{l}\text { - Hospitalisation et anesthésie obligatoires } \\
\text { - Complications opératoires (0-3\%) } \\
\text { - Synéchies utérines (curetages itératifs, contexte septique) (NP 3) }\end{array}$ \\
\hline Misoprostol & $\begin{array}{l}\text { - Prise en charge ambulatoire } \\
\text { - Réduction du nombre de gestes chirurgicaux (NP 2) } \\
\text { - Risques infectieux et hémorragiques non augmentés (NP 1) } \\
\text { - Facilitation du geste chirurgical si rétention secondaire } \\
\text { - Risque de consultation en urgence identique (NP 2) }\end{array}$ & $\begin{array}{l}\text { - Efficacité inférieure à celle de la chirurgie }(>80 \% \text { b }) \text { (NP 1) } \\
\text { - Variable selon les modalités d'administration (voie orale, vaginale } \\
\text { sublinguale, posologie unique ou répétée), le délai accordé (24 } \\
\text { heures-15 jours) et le type de FC (retardée/incomplète) (NP 2) } \\
\text { - Durée des saignements plus longue, douleurs plus importantes (NP 2) } \\
\text { - Hospitalisations non programmées plus fréquentes (NP 2) }\end{array}$ \\
\hline Expectative & $\begin{array}{l}\text { - Taux de succès élevéa en cas de FC en cours ou incomplète (> } 75 \text { \%) } \\
\text { - Evite les complications et les coûts du traitement chirurgical } \\
\text { - Risques infectieux et hémorragiques non augmentés (NP 2) }\end{array}$ & $\begin{array}{l}\text { - Délai d'expulsion variable (3 jours-6 semaines), souvent inacceptable } \\
\text { pour les patientes (NP 2) } \\
\text { - Augmentation du nombre de consultations et de gestes chirurgicaux } \\
\text { en urgence (NP 2) }\end{array}$ \\
\hline
\end{tabular}


Tableau 3 : Principales études ayant évalué le misoprostol dans le cadre de l'induction du travail en cas de mort foetale au-delà de 14 SA.

Main Studies that evaluated misoprostol under induction of labor only in cases of fetal death beyond 14 weeks.

\begin{tabular}{|c|c|c|c|c|c|c|c|c|}
\hline \multirow{4}{*}{ Types d'études } & \multirow{4}{*}{$\begin{array}{l}\text { Age gestationnel } \\
\text { d'inclusion (SA) } \\
\text { (moyenne ou } \\
\text { médiane*) }\end{array}$} & \multicolumn{3}{|c|}{ Misoprostol } & \multirow{3}{*}{$\begin{array}{c}\text { Intervalle } \\
\text { induction-expulsion } \\
\text { (moyenne ou }\end{array}$} & \multicolumn{2}{|c|}{ Taux de succès } & \multirow{4}{*}{ Remarques } \\
\hline & & \multicolumn{3}{|c|}{ Groupe étude/groupe témoin } & & \multicolumn{2}{|c|}{ à 24 et 48 heures } & \\
\hline & & Voie & Dose & Intervalle & & $(\%)$ & & \\
\hline & & & $(\mu \mathrm{g})$ & (heures) & médiane* en heures) & & & \\
\hline \multicolumn{9}{|l|}{ Misoprostol seul } \\
\hline Eng $1997[54]$ & $13-26(18,6)$ & Vaginal & 200 & 3 & $\mathrm{NC}$ & 84 & $\mathrm{NC}$ & \\
\hline Randomisée, $\mathrm{N}=25$ & & Géméprost & $1 \mathrm{mg}$ & 3 & & 68 & & \\
\hline Nakintu 2001 [55] & $18-40$ & Vaginal & 50 & 6 & 12,4 & $\mathrm{NC}$ & & Augmentation des doses de $50 \mu \mathrm{g}$ toutes les \\
\hline \multirow[t]{3}{*}{ Randomisée, $\mathrm{N}=60$} & & Oxytocine & & & & & & 6 heures \\
\hline & & & & & & & & Taux de succès entre 23 et $28 \mathrm{SA}: 15$ \\
\hline & & & & & & & & versus 25 heures \\
\hline Chitttacharoen 2003 [56] & Plus de 16 & Oral & 400 & 4 & $13,9 \pm 5,6$ & 92,5 & 100 & Taux de succès à 24 heures : $100 \%$ entre 17 \\
\hline Randomisée, $\mathrm{N}=80$ & $(23,8$ et 22,7$)$ & Vaginal & 200 & 12 & $18,9 \pm 10,4$ & 67,5 & 100 & et $26 \mathrm{SA}$ \\
\hline Fadalla 2004 [57] & $13-28$ & Oral & 100 & 4 & $14,9 \pm 3,4$ & $\mathrm{NC}$ & & Recours à l'oxytocine 31 versus $11 \%(\mathrm{p}=$ \\
\hline Randomisée, $\mathrm{N}=70$ & $(21,6$ et 25,5$)$ & Vaginal & 100 & 4 & $10,8 \pm 2,8$ & $\mathrm{NC}$ & & $0,04)$, à une chirurgie complémentaire 26 \\
\hline & & & & & & & & versus $6 \%(\mathrm{p}=0,02)$ \\
\hline Nyende 2004 [58] & $\mathrm{NC}$ & Oral & 200 & 6 & $21,4 \pm 14$ & 100 & & Recours à l'oxytocine 55 versus $20 \%$ (p \\
\hline
\end{tabular}




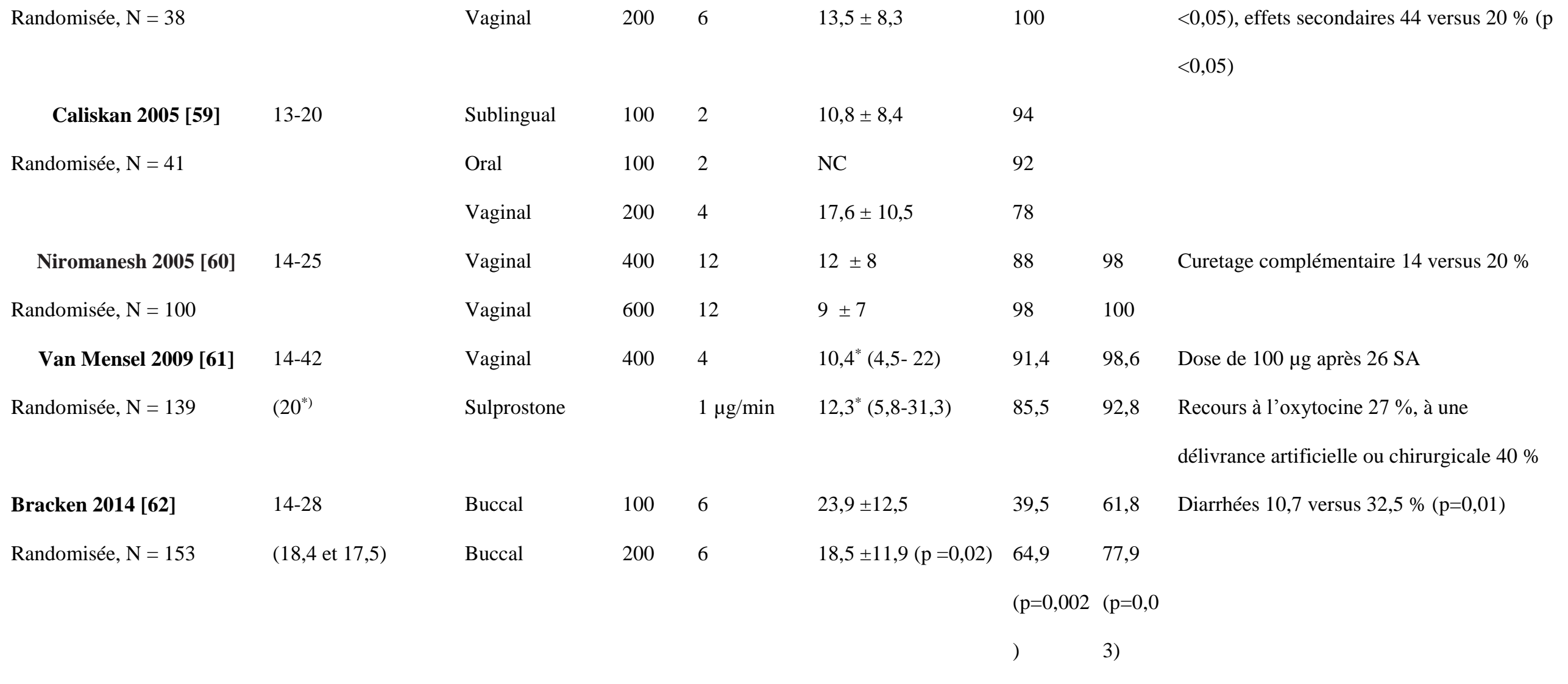

\section{Mifépristone (dose et délai) + misoprostol}

\begin{tabular}{|c|c|c|c|c|c|c|}
\hline $\begin{array}{ll}\text { Wagaarachchi 2002 [63] } 24-41 \\
\end{array}$ & Vaginal & 200 & $8,5^{*}(0,5-76)$ & 87,5 & 96 & $200 \mu \mathrm{g}$ avant $34 \mathrm{SA}, 100 \mu \mathrm{g}$ après $34 \mathrm{SA}$ \\
\hline Prospective, non randomisée, $\left(32,4^{*}\right)$ & Oral & 100 & & & & Diminution du délai induction expulsion \\
\hline $\mathrm{N}=96$ & & & & & & avec l'augmentation de l'âge gestationnel ( \\
\hline $600 \mathrm{mg}, 24-48$ heures) & & & & & & $\mathrm{p}=0,04) 1$ \\
\hline
\end{tabular}


Fairley 2005 [64]

Prospective, non randomisée

$\mathrm{N}=49$

Vaginal

(200 mg, 36-48 heures)

Sharma 2011 [65]

Prospective, $\mathrm{N}=40$

Oral

50 à

100

(200 mg, 36 heures)

$$
7^{*}(1,5-29,5)
$$

NC

NC

50

$10,2^{*}(1,5-20)$

SA : semaines d'aménorrhée ; NC : non communiqué ; * : médiane (déviations standards) 
Figure 1 : Algorithme décisionnel en cas de FC du premier trimestre (grossesse arrêtée et FC incomplète). Decision algorithm in case of an early miscarriage.

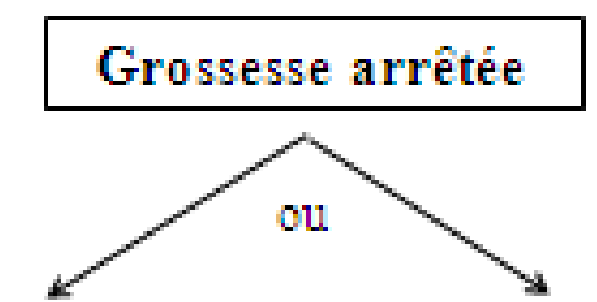

Misoprostol

$800 \mu \mathrm{g}$ (voie vaginale)

Grade B

\section{Fausse couche incomplete}

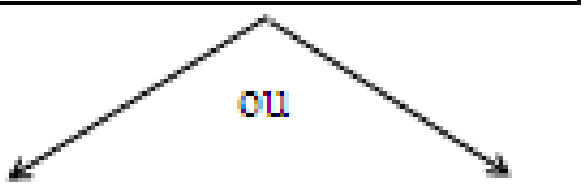

Expectative

Grade A 